\title{
Two-dimensional assembly and local redox-activity of molecular hybrid structures in an electrochemical environment
}

\author{
Z. Li,${ }^{a}$ B. Han, ${ }^{a}$ G. Meszaros $, \dagger^{a}{ }^{\text {I. Pobelov }},{ }^{a}$ Th. Wandlowski, ${ }^{a}$ A. Błaszczyk $\ddagger^{b}$ \\ and M. Mayor $\$^{b}$
}

Received 10th May 2005, Accepted 30th June 2005

First published as an Advance Article on the web 15th September 2005

DOI: $10.1039 / \mathbf{b 5 0 6 6 2 3 a}$

\begin{abstract}
The self-assembly and redox-properties of two viologen derivatives, $N$-hexyl- $N^{\prime}$ (6-thiohexyl)-4,4'-bipyridinium bromide (HS-6V6-H) and $N, N^{\prime}$-bis(6-thiohexyl)-4,4'bipyridinium bromide (HS-6V6-SH), immobilized on $\mathrm{Au}(111)-(1 \times 1)$ macro-electrodes were investigated by cyclic voltammetry, surface enhanced infrared spectroscopy (SEIRAS) and in situ scanning tunneling microscopy (STM). Depending on the assembly conditions one could distinguish three different types of adlayers for both viologens: a low coverage disordered and an ordered "striped" phase of flat oriented molecules as well as a high coverage monolayer composed of tilted viologen moieties. Both molecules, HS-6V6-H and HS-6V6-SH, were successfully immobilized on Au(poly) nano-electrodes, which gave a well-defined redox-response in the lower pA-current range. An in situ STM configuration was employed to explore electron transport properties of single molecule junctions $\mathrm{Au}(\mathrm{T})|\mathrm{HS}-6 \mathrm{~V} 6-\mathrm{SH}(\mathrm{HS}-6 \mathrm{~V} 6-\mathrm{H})| \mathrm{Au}(\mathrm{S})$. The observed sigmoidal potential dependence, measured at variable substrate potential $E_{\mathrm{S}}$ and at constant bias voltage $\left(E_{\mathrm{T}}-E_{\mathrm{S}}\right)$, was attributed to electronic structure changes of the viologen moiety during the one-electron reduction/re-oxidation process $\mathrm{V}^{2+} \leftrightarrow \mathrm{V}^{+} \cdot$. Tunneling experiments in asymmetric, STM-based junctions $\mathrm{Au}(\mathrm{T})-\mathrm{S}-6 \mathrm{~V} 6-\mathrm{H} \mid \mathrm{Au}(\mathrm{S})$ revealed current $\left(i_{\mathrm{T}}\right)$-voltage $\left(E_{\mathrm{T}}\right)$ curves with a maximum located at the equilibrium potential of the redox-process $\mathrm{V}^{2+} \leftrightarrow \mathrm{V}^{+} \cdot$ The experimental $i_{\mathrm{T}}-E_{\mathrm{T}}$ characteristics of the HS-6V6-H-modified tunneling junction were tentatively attributed to a sequential two-step electron transfer mechanism.
\end{abstract}

\section{Introduction}

As the miniaturization of electronic components approaches the nanometer scale, new concepts to tailor structure, functionality and fabrication strategies are essential to overcome the fundamental physical and economic limitations of conventional inorganic silicon technology. ${ }^{1}$ Bottom up (self-) assembly of well-defined nanoscale building blocks, such as molecules, ${ }^{2}$ quantum dots, ${ }^{3}$ and nanowires, ${ }^{4}$ having key properties controlled by size, morphology and chemical composition represent an attractive alternative. The idea of building an electronic device using individual molecules was first proposed by Aviram and Ratner. ${ }^{5}$ The molecular approach bears several unique opportunities: (1) custom-design of nanoscale molecular units, (2) high reproducibility due to

\footnotetext{
${ }^{a}$ Institute of Surfaces and Interfaces ISG 3 and cni-Center of Nanoelectronic Systems for Information Technology, Research Center Jülich GmbH, D-52425, Jülich, Germany. E-mail: th.wandlowski@fz-juelich.de

${ }^{b}$ Institute for Nanotechnology, Research Center Karlsruhe GmbH, P. O. Box 3640, D-76021, Karlsruhe, Germany.E-mail: marcel.mayor@int.fzk.de
}

$\uparrow$ Home address: Institute of Materials and Environmental Chemistry, Chemical Research Center of the Hungarian Academy of Sciences, Pusztaszeri ut 59-67, H-1025 Budapest, Hungary.

t Home address: Faculty of Commodity Science, Al. Niepodleglości 10, 60-967 Poznań, Poland.

$\S$ Also: Department of Chemistry, University of Basel, St. Johanns-Ring 19, CH-4056 Basel, Switzerland. 
self-organization and recognition properties of the molecular building blocks, (3) irhiplenterindlationiroof localized functions such as charge transfer, $\pi-\pi$ stack conductivity or molecular rectification, etc. ${ }^{6,7}$

The development of several experimental techniques, including mechanically controlled break junctions, ${ }^{8-10}$ nanopores, ${ }^{11,12}$ nanowires,${ }^{4}$ crossed wires, ${ }^{13}$ solid metal film ${ }^{14}$ and mercury drop junctions, ${ }^{15}$ and various scanning probes $(\mathrm{SPM})^{2,16-26}$ have enabled the ability to explore electrical properties of tailored individual molecules and/or molecular assemblies attached to conducting substrates. In SPM and surface nano-electrochemistry, the free or cluster tagged molecules ${ }^{20,23}$ are typically in direct contact with one metal electrode. Bridging of individual molecules between a gold STM tip and a gold substrate was recently achieved in solution. ${ }^{25-28}$ The statistical analysis of repeatedly created junctions provided access to single molecule conductivities.

Several groups explored solid state assemblies (metallmoleculelmetal junctions) with redoxcenters, exhibiting low-lying discrete HOMO respective LUMO levels, under ex situ or low temperature conditions (ambient, UHV) and discovered phenomena such as negative differential resistance (NDR), ${ }^{29}$ rectification, ${ }^{6}$ amplification, ${ }^{30-33}$ stable switching and memory effects. ${ }^{34,35}$ These observations were attributed to molecular (redox) energy states brought to resonance with the Fermi levels of the enclosing electrodes by the applied bias voltage or by nuclear configuration fluctuations (dominant at RT), respective vibrational relaxations (dominant at cryogenic temperatures). Examples of functional molecules used in these studies are $2^{\prime}$-amino-4, $4^{\prime}$-di(ethynylphenyl)$5^{\prime}$-nitro-1-benzene thiolate, ${ }^{11}$ metalorganic $\mathrm{Co}^{2+}$ and $\mathrm{Fe}^{2+}$-terpyridine complexes, ${ }^{36}$ oligothiophenes, ${ }^{32}$ p-phenylenvinylen oligomers ${ }^{31}$ and azurine. ${ }^{33}$

First examples for switching and rectification under electrochemical (and closely related) conditions were reported for porphyrins, ${ }^{19,37}$ phthalocyanines, ${ }^{38,39}$ metal proteins ${ }^{21,40,41}$ and $\mathrm{Ru}-$ modified alkanethiols ${ }^{15}$ deposited on HOPG or gold electrodes, gold nanoparticles tethered to a gold substrate with an alkylviologen redox group, ${ }^{20}$ as well as for nanometer-size junctions bridged with heptaaniline oligomers ${ }^{28}$ or polyaniline. ${ }^{42}$ The molecular energetic states are coupled to the electrolyte environment, and to the metallic interfaces, characterized by electronic transmission ("coupling") coefficients. The following scenarios of interfacial electron transfer in a nanoscale electrochemical metal|redox moleculelmetal configuration can be envisaged: ${ }^{21}$ (1) superexchange via off-resonance levels, ${ }^{43}$ (2) resonance mode, ${ }^{44}$ (3) coherent two-step electron transfer, ${ }^{21,43}$ (4) sequential two-step electron transfer. ${ }^{21,43,45}$

Working in an electrochemical environment has the advantage that two potential differences can be controlled individually: the bias voltage between two working electrodes, and the potential drop between one working electrode and a reference electrode. The latter may be considered as a "gate electrode". The current flow to the external circuit is assisted by a fourth electrode, the counter electrode (cf. 21). The idea of an electrochemical gate for applications in molecular electronics was introduced by Schönenberger et al. ${ }^{46}$ and McEuen et al. ${ }^{47}$ The field in the electrochemical double layer (EDL) is close to the gate field required to significantly change the current through a molecule, according to first principle calculations by di Ventra et al. ${ }^{48}$

Motivated by the pioneering work of the Liverpool group on single molecule conductivity ${ }^{20,26,27}$ we report in this contribution a comparative study on the redox-activity of $N$-hexyl- $N^{\prime}$-(6thiohexyl)-4,4'-bipyridinium bromide (HS-6V6-H) and $N, N^{\prime}$-bis(6-thiohexyl)-4,4'-bipyridinium bromide (HS-6V6-SH) immobilized and addressed on gold electrodes in an electrochemical environment. The tailored molecules are composed of a redox-active center, the viologen group, flexible alkyl-spacer units and one or two terminal SH anchor groups. Our approach combines classical and low current voltammetry (sub-picoampere resolution) with surface enhanced infrared spectroscopy (SEIRAS), ${ }^{49}$ in situ scanning tunneling microscopy (STM) and spectroscopy (STS). ${ }^{50}$ The main goal of the present paper is focused on measurements of electron transfer with redoxactive, thiol-functionalized viologens as they are addressed by the electrochemical potential to explore relations between molecular and electronic structure in macroscale and nanoscale molecular assemblies approaching the single molecule level.

The redox-active viologens occur in three different oxidation states $\mathrm{V}^{2+} \leftrightarrow \mathrm{V}^{+} \cdot$ and $\mathrm{V}^{+} \cdot \leftrightarrow$ $\mathrm{V}{ }^{51,52}$ They have been incorporated as "backbone"-components in self-assembled monolayers, ${ }^{53-60}$ or in a nanometer-scale electronic switch ${ }^{20,26}$ and various functional materials. ${ }^{61}$ The self-assembly and redox-functionality of thiol-functionalized viologens on gold macro-electrodes was investigated by cyclic voltammetry, ${ }^{53,54,56-60}$ quartz crystal microbalance, ${ }^{53} \mathrm{IR}^{62,63}$ and Raman spectroscopy, ${ }^{62}$ electro-reflectance, ${ }^{57,59}$ ex situ $\mathrm{STM}^{20,63}$ and STS. ${ }^{26,63}$ 


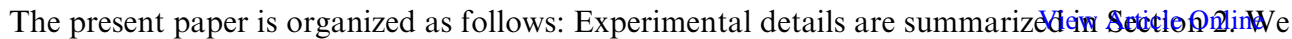
report electrochemical studies on $\mathrm{Au}$ (111) single crystal macro-electrodes and gold nano-electrodes on the self-assembly and redox-activity of HS-6V6-H and HS-6V6-SH employing cyclic voltammetry, in situ SEIRAS and STM. The final paragraph addresses an attempt to explore single molecule electron transport and electron tunneling at a potential-controlled electrode/electrolyte interface.

\section{Experimental}

\subsection{Synthesis}

The synthetic strategy to the viologen derivatives HS-6V6-H (1) and HS-6V6-SH (2) is based on the nucleophilic substitution reaction between pyridines and terminal alkyl bromides (Scheme 1). ${ }^{64}$

HS-6V6-H (1). The viologen core was first mono-functionalized with a hexyl chain. 4,4'Bipyridine and the corresponding 1-bromo- $n$-hexane 3 have been heated to $90{ }^{\circ} \mathrm{C}$ in chloroform in a pressure tube. The crude products were dissolved in dichloromethane and separated from the insoluble doubly substituted viologen derivatives by filtration to afford the desired mono-substituted viologen 4 in reasonable yields of $78 \%$. The nucleophilic terminal sulfur groups are masked as thioacetates. ${ }^{65}$ The required bifunctional hexyl chain with a bromine atom at one end and an acetyl-protected sulfur at the opposite end 7 has been synthesized from the bromohexene $\mathbf{6}$ and thioacetic acid in the presence of a radical source (yield 81\%). ${ }^{66}$ Bromohexene 6 has been converted to 7 in good yields $(81 \%)$ by refluxing in toluene with thioacetic acid and 1,1'-azobis(cyclohexanecarbonitrile). The acetyl protected hexyl bromide 7 and the mono-hexylviologen derivative 4 have been heated to $110{ }^{\circ} \mathrm{C}$ in dimethylformamide (DMF) to form the acetyl-protected target structure 5 in very good yields $(97 \%)$. In variation of the literature procedure ${ }^{65}$ we replaced acetylchloride by acetylbromide to form in situ $\mathrm{HBr}$ in methanol under an argon atmosphere to deprotect the acetyl groups of 5. HS-6V6-H (1) has been isolated as the pure bromide salt (yield $92 \%$ ) as confirmed by elemental analysis.

HS-6V6-SH (2). 2.3 Equivalents of the terminally acetyl protected thiol-functionalized hexylbromide 7 have been used to substitute both nitrogens of 4,4'-bipyridine. The acetyl protected target structure 8 has been isolated in good yields $(81 \%)$ after crystallization from ethanol. The acetylprotected viologen dithiol $\mathbf{8}$ was dissolved in methanol and an excess of acetylbromide was added at $-78{ }^{\circ} \mathrm{C}$ to afford the desired viologen dithiol 2 after stirring at room temperature (yield $95 \%$ ).

$\boldsymbol{N}$-Hexyl- $\boldsymbol{N}^{\prime}$-(6-thiohexyl)-4,4'-bipyridinium bromide, HS-6V6-H (1). Yellow solid. Mp 274$27{ }^{\circ} \mathrm{C} ; \delta_{\mathrm{H}}\left(300 \mathrm{MHz} ; \mathrm{MeOD} ; \mathrm{Me}_{4} \mathrm{Si}\right) 0.93\left(\mathrm{t}, 3 \mathrm{H}, \mathrm{CH}_{3}, J=6.9 \mathrm{~Hz}\right), 1.30-1.70(\mathrm{~m}, 12 \mathrm{H}), 2.04-$ $2.20(\mathrm{~m}, 4 \mathrm{H}), 2.52\left(\mathrm{t}, 2 \mathrm{H}, \mathrm{SCH}_{2}, J=6.4 \mathrm{~Hz}\right), 4.77(\mathrm{td}, 4 \mathrm{H}, J=2.1, J=6.8 \mathrm{~Hz}), 8.70(\mathrm{~d}, 4 \mathrm{H}, J=6.7$ $\mathrm{Hz}), 9.30(\mathrm{~d}, 4 \mathrm{H}, J=6.1 \mathrm{~Hz}) ; \delta_{\mathrm{C}}\left(75 \mathrm{MHz}\right.$; MeOD; $\left.\mathrm{Me}_{4} \mathrm{Si}\right) 14.28,23.49,24.76,26.65,26.91,28.67$,
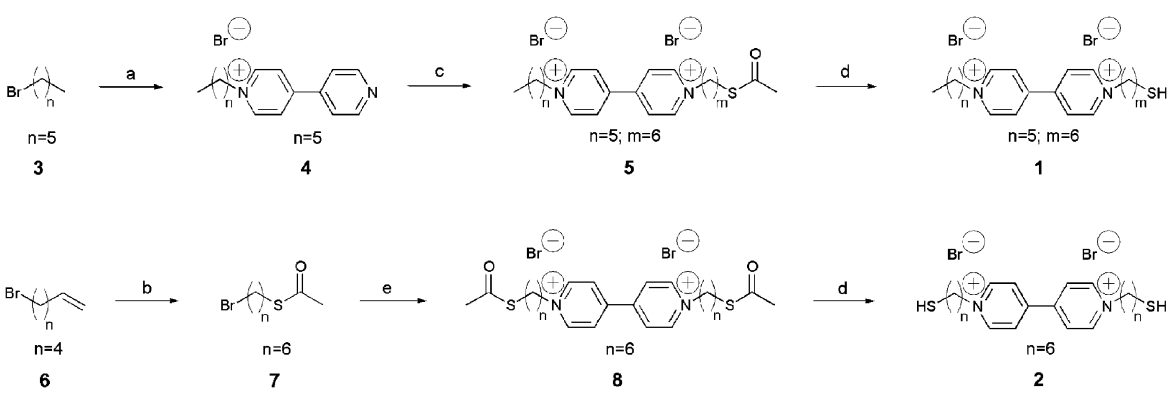

Scheme 1 Synthesis of the viologen monothiol 1 and dithiol 2. (a) 4,4'-Bipyridine, $\mathrm{CHCl}_{3}, 90{ }^{\circ} \mathrm{C}, 16 \mathrm{~h}$; (b) $\mathrm{CH}_{3} \mathrm{COSH}, 1,1^{\prime}$-azobis(cyclohexanecarbonitrile), toluene, reflux, $1 \mathrm{~h}$; (c) $\mathrm{Br}\left(\mathrm{CH}_{2}\right)_{6} \mathrm{SCOCH}_{3}, \mathrm{DMF}, 110{ }^{\circ} \mathrm{C}$, $24 \mathrm{~h}$; (d) $\mathrm{AcBr}, \mathrm{MeOH},-78{ }^{\circ} \mathrm{C}$ to rt, argon atmosphere, $3 \mathrm{~h}$; (e) 4,4'-bipyridine, DMF, $110{ }^{\circ} \mathrm{C}, 24 \mathrm{~h}$. 
32.32, 32.44, 32.54, 34.78, 63.20, 63.30, 128.33, 147.10, 151.31; Elemental analy\$is vealcticleolonlfior $\mathrm{C}_{22} \mathrm{H}_{34} \mathrm{Br}_{2} \mathrm{~N}_{2} \mathrm{~S}$ : C 50.97, H 6.61, N 5.40, found: C 50.69, H 6.52, N 5.68; $\mathrm{m} / z$ (MALDI TOF) $357.5839\left[\mathrm{M}^{+}-2 \mathrm{Br}\right]$.

$\boldsymbol{N}, \boldsymbol{N}^{\prime}$-Bis(6-thiohexyl)-4,4'-bipyridinium bromide, HS-6V6-SH (2). Yellow solid (95\%). Mp 263$265^{\circ} \mathrm{C} ; \delta_{\mathrm{H}}\left(300 \mathrm{MHz} ; \mathrm{MeOD} ; \mathrm{Me}_{4} \mathrm{Si}\right) 1.38-1.58(\mathrm{~m}, 8 \mathrm{H}), 1.64(\mathrm{q}, 4 \mathrm{H}, J=7.0 \mathrm{~Hz}), 2.11(\mathrm{q}, 4 \mathrm{H}, J=$ $7.6 \mathrm{~Hz}), 2.51\left(\mathrm{t}, 4 \mathrm{H}, J=6.8 \mathrm{~Hz}, \mathrm{SCH}_{2}\right), 4.75\left(\mathrm{t}, 4 \mathrm{H}, J=7.5 \mathrm{~Hz}, \mathrm{NCH}_{2}\right), 8.69(\mathrm{~d}, 4 \mathrm{H}, J=6.6 \mathrm{~Hz})$, $9.29(\mathrm{~d}, 4 \mathrm{H}, J=6.6 \mathrm{~Hz}) ; \delta_{\mathrm{C}}\left(75 \mathrm{MHz} ; \mathrm{MeOD} ; \mathrm{Me}_{4} \mathrm{Si}\right) 25.65,27.45,29.60,33.21,35.60,64.10$, 129.31, 148.10, 152.32; Elemental analysis calcd. (\%) for $\mathrm{C}_{22} \mathrm{H}_{34} \mathrm{Br}_{2} \mathrm{~N}_{2} \mathrm{~S}_{2}$ : C 48.00, H 6.23, N 5.09, found: C 48.27, H 6.49, N 5.05; $m / z$ (MALDI TOF) $390.0631\left[\mathrm{M}^{+}-2 \mathrm{Br}\right]$.

\subsection{Electrolyte solutions, electrodes and electrochemical measurements}

The electrolyte solutions were prepared with Milli-Q water (18 M $\Omega, 2$ ppb TOC), $\mathrm{KClO}_{4}($ Fluka puriss p.a., twice recrystallized from water), $\mathrm{HCl}$ (suprapure Merck), $\mathrm{KOH}$ (suprapure Merck), $\mathrm{Na}_{2} \mathrm{HPO}_{4} \cdot 2 \mathrm{H}_{2} \mathrm{O}$ (Fluka, puriss p.a.), $\mathrm{NaH}_{2} \mathrm{PO}_{4} \cdot \mathrm{H}_{2} \mathrm{O}$ (Fluka, BioChem Ultra) and ethanol (p.a., $\mathrm{KMF}$ ). All electrolytes were deaerated with argon before and during the experiments. The measurements were carried out at $20 \pm 0.5{ }^{\circ} \mathrm{C}$. The glassware was cleaned either in caroic acid or in a $1: 1$ mixture of hot $\mathrm{H}_{2} \mathrm{SO}_{4}\left(95-97 \%\right.$, pro-analysis, Merck) and $\mathrm{HNO}_{3}(65 \%$ purissimum, Riedel-de-Haen), followed by extended rinsing with Milli-Q water.

Single crystal $\mathrm{Au}(111)$ electrodes were cylinders (EC, $4 \mathrm{~mm}$ height and $4 \mathrm{~mm}$ diameter) or discs (STM, $2 \mathrm{~mm}$ height and $10 \mathrm{~mm}$ diameter). They were flame-annealed with a hydrogen torch at red heat, and then cooled in high purity argon. Contact with the electrolyte was always established under potential control. Before modification with viologens island-free $\mathrm{Au}(111)-(1 \times 1)$ surfaces were prepared by immersing a freshly flame-annealed electrode under potential control at $0.50 \mathrm{~V}$ ( $v s$. SCE) into deaerated $0.1 \mathrm{M} \mathrm{HCl}$. After a waiting time of $60 \mathrm{~s}$, the electrode was removed and thoroughly rinsed with Milli-Q water. ${ }^{67}$

Gold nano-electrodes for electrochemical and STM experiments were prepared by etching a 0.25 $\mathrm{mm}$ diameter gold wire in a $1: 1$ mixture of ethanol and $36 \% \mathrm{HCl}^{68}$ and subsequent coating with polyethylene. The uncovered areas of these electrodes were typically 1 to $15 \times 10^{-12} \mathrm{~m}^{2}$. ${ }^{69}$

The preparation and characterization of quasi-single crystalline $\mathrm{Au}(111-25 \mathrm{~nm})$ film electrodes used as substrate material in all SEIRAS experiments has been described previously in ref. 49.

Electrochemical experiments with macro-electrodes were carried out with a custom-made set-up described in ref. 70. A home-made low current potentiostat (resolution $<10 \mathrm{fA}$ ) has been developed for the studies with nano-electrodes. ${ }^{69}$ All potentials in this paper are quoted with respect to a saturated calomel electrode (SCE). Contact to the electrolyte was always established under strict potential control.

\subsection{Electrode modification}

Three different protocols have been developed to prepare gold electrodes modified with HS-6V6-H or HS-6V6-SH: (1) Low coverage disordered adlayers were obtained after immersion of a freshly prepared $\mathrm{Au}(111)-(1 \times 1)$ electrode into a $50 \mu \mathrm{M}$ deoxygenated ethanolic solution of the respective viologen derivative at room temperature for $1 \mathrm{~min}$. (2) Low coverage ordered adlayers ("striped phases", $c f$. paragraph 3.4) were prepared by exposure of the gold substrates at room temperature to $50 \mu \mathrm{M}$ deoxygenated ethanolic viologen-containing solution for $2 \mathrm{~min}$, and subsequent annealing in pure ethanol at $70{ }^{\circ} \mathrm{C}$ for $12 \mathrm{~h}$. (3) High coverage monolayers were obtained by immersion of the gold substrates into $1 \mathrm{mM}$ ethanolic solution followed by thermal annealing in ethanol at $70{ }^{\circ} \mathrm{C}$ for $12 \mathrm{~h}$. The temperature treatment of the samples was carried out in closed containers, which were deoxygenated. After incubation, the samples were removed from the solutions, rinsed with ethanol and carefully dried in a stream of argon.

\subsection{Spectroelectrochemical set-up}

The in situ SEIRAS experiments were carried out with a Bruker IF 66v/s Fourier Transform Spectrometer synchronized with a Heka PG 310 potentiostat employing a vertical 
spectroelectrochemical cell in Kretschmann ATR-configuration and p-polarizedVinffratredideadindition from a globar source. ${ }^{49}$ The working electrode was a quasi-single crystalline Au(111-25 nm) film electrode prepared by electron beam evaporation of gold onto the (111) plane of a silicon hemisphere, and subsequent electrochemical annealing. ${ }^{49}$ The spectroelectrochemical measurements were carried out under potential sweep or potential step conditions. In potential sweep experiments $\left(10\right.$ or $50 \mathrm{mV} \mathrm{s}^{-1}$ ), the spectrometer was operated in the slow scan mode. Typically, 80 sequentially acquired interferograms were co-added into each single beam spectrum. The spectra were plotted in absorbance units defined as $A=-\log \left(I / I_{\mathrm{o}}\right)$, where $I$ and $I_{\mathrm{o}}$ represent the intensities of the reflected radiation at the sample and the reference potentials, respectively. The complete description of the spectroelectrochemical ATR-SEIRAS experiment and of the sample preparation is given in ref. 49 .

\subsection{STM and STS measurements}

The STM and STS experiments were carried out with a Molecular Imaging Pico-SPM which was positioned in a hermetically sealed container to prevent oxygen exposure of the samples. The STM tips were electrochemically etched tungsten or gold wires $(0.25 \mathrm{~mm}$ diameter $)$ coated with polyethylene. Platinum wires served as quasi-reference and counter electrodes. Experiments in neutral electrolytes were carried out with silver wires as quasi-reference electrodes. All STM experiments were performed at room temperature in constant current mode with tunneling currents ranging between 3 and $200 \mathrm{pA}$.

Local electron transfer characteristics of Au(T)|HS-6V6-SH(HS-6V6-H) $\mid \mathrm{Au}(\mathrm{S})$ junctions were explored by combining current distance measurements with in situ STM imaging. ${ }^{50}$ A gold tip (T) coated with polyethylene (free area of 1 to $15 \times 10^{-12} \mathrm{~m}^{2}$ ) was brought to a predefined $x y z$-position and held at fixed bias $\left(E_{\mathrm{T}}-E_{\mathrm{S}}\right)$. The distance $z_{\mathrm{O}}$ to the adsorbate-covered gold substrate (S) was fixed choosing a tunneling setpoint current $i_{\mathrm{o}}$ of 50 or $100 \mathrm{pA}$. After stabilization, the $z$-piezo feedback was disabled temporarily. The tip approached the surface up to $z_{\mathrm{o}}-\Delta z_{\mathrm{a}}$ with maximum rate, was held at this position for $100 \mathrm{~ms}$, and subsequently retracted to $z_{\mathrm{o}}+\Delta z_{\mathrm{r}}$. The individual pulling traces were recorded (2000 data points, current range between $1 \mathrm{pA}$ and $10 \mathrm{nA}$ or, occasionally, between $10 \mathrm{pA}$ and $100 \mathrm{nA}$ ). Typical values of $\Delta z_{\mathrm{a}}$ and $\Delta z_{\mathrm{r}}$ were $1.1 \mathrm{~nm}$ and $2 \mathrm{~nm}$. $i_{\mathrm{o}}$ and $\Delta z_{\mathrm{a}}$ were carefully selected to ensure strong tip-adsorbate interactions, but not to drive the tip into the substrate surface. ${ }^{25,28,50}$ The strategy allowed the spontaneous formation of molecular bridges between the gold STM tip and the substrate surface, ${ }^{27}$ which were subsequently broken upon retraction. After enabling the $z$-feedback again, with the preset values of $i_{\mathrm{o}}$, the surface was inspected by STM-imaging in constant current mode.

Current-voltage spectroscopy was carried out with viologen-modified gold tips. The redoxactivity of these tips was tested by cyclic voltammetry. After mounting in the STM set-up the system was stabilized for several hours in a controlled argon atmosphere. The tunneling regime was established with set-point currents $i_{\mathrm{o}}$ ranging between $30 \mathrm{pA}$ and $200 \mathrm{pA}$ to preserve the integrity of the viologen derivative in the gap. After equilibration, the $z$-piezo feedback was switched off, and several current voltage traces were recorded at fixed bias $\left(E_{\mathrm{T}}-E_{\mathrm{S}}\right)$ by sweeping simultaneously the potentials of tip $\left(E_{\mathrm{T}}\right)$ and substrate $\left(E_{\mathrm{S}}\right)$ with $2 \mathrm{~V} \mathrm{~s}^{-1}$. We recorded individual single scans with 1000 data points. The cycle was repeated after stabilization at the preset values of $i_{\mathrm{o}}$.

\section{Results and discussion}

\subsection{Cyclic voltammetry with single crystal macro-electrodes}

Fig. 1A shows a typical first scan voltammogram of an $\mathrm{Au}(111)-(1 \times 1)$ electrode modified with a monolayer of HS-6V6-H, bromide salt, in $0.05 \mathrm{M} \mathrm{KClO}_{4}$ adjusted to $\mathrm{pH}=10$ by addition of $\mathrm{KOH}$. The solution resistance was carefully compensated by applying a positive feedback. The adlayer was prepared by immersion of the gold electrode in $1 \mathrm{mM}$ ethanolic solution of HS-6V6-H followed by thermal annealing at $70{ }^{\circ} \mathrm{C}$ for $12 \mathrm{~h}$ (protocol 3). Contact with the electrolyte was established at $E=$ $-0.700 \mathrm{~V}$. The electrode potential was first scanned in the positive direction. The adlayer capacitance was estimated to be $8.8 \mu \mathrm{F} \mathrm{cm} \mathrm{cm}^{-2}$ in $-0.200 \mathrm{~V}<E<-0.300 \mathrm{~V}$. This value is rather large compared to alkanethiols ${ }^{71}$ and indicates the incorporation of water molecules or ions into the film. Subsequent potential excursion reveals three characteristic pairs of current peaks labeled $\mathrm{P} 1 / \mathrm{P}^{\prime}, \mathrm{P} 2 / \mathrm{P} 2^{\prime}$ and $\mathrm{P} 3 / \mathrm{P}^{\prime}$. Restricting the potential range to $E \geq-1.050 \mathrm{~V}$ results in two pairs 


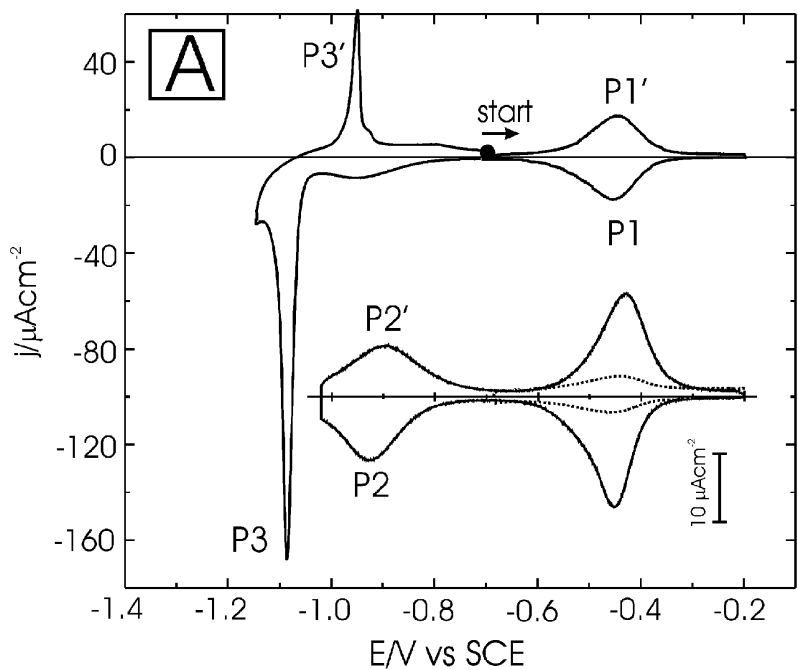

View Article Online

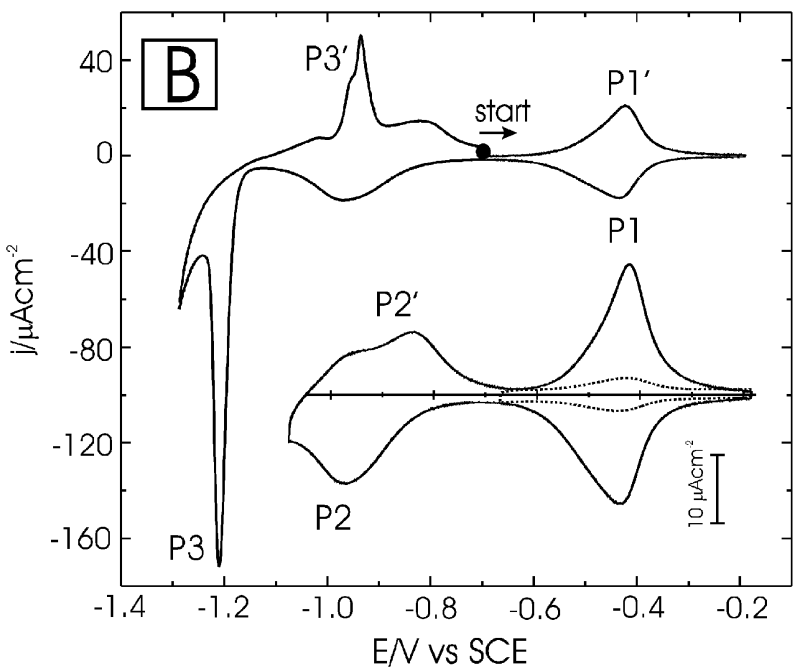

Fig. 1 First scan cyclic voltammograms (solid lines) of $\mathrm{Au}(111)-(1 \times 1)$ electrodes in $0.05 \mathrm{M} \mathrm{KClO}_{4}, \mathrm{pH}$ adjusted to 10 with $\mathrm{KOH}$, modified with HS-6V6-H (A) and HS-6V6-SH (B) according to protocol 3, sweep rate $50 \mathrm{mV} \mathrm{s}^{-1}$. The characteristic current peaks are labeled $\mathrm{P} 1 / \mathrm{P}^{\prime}, \mathrm{P} 2 / \mathrm{P} 2^{\prime}$ and $\mathrm{P} 3 / \mathrm{P} 3^{\prime}$. The insets show first-scan voltammograms recorded in a limited potential range for high coverage (solid lines, protocol 3 ) and low coverage (dotted lines, protocol 2) viologen adlayers.

of well-defined current peaks (Fig. 1A). $\mathrm{P} 1(E=-0.440 \mathrm{~V})$ and $\mathrm{P}^{\prime}(E=-0.428 \mathrm{~V})$ are assigned to the one-electron reaction $\mathrm{V}^{2+} \leftrightarrow \mathrm{V}^{+\cdot} \cdot 51,52$ The peak-to-peak separation is rather constant up to $2 \mathrm{~V} \mathrm{~s}^{-1}$, and the peak height scales linearly with the scan rate. The peak FWHM (P1, $\left.\mathrm{P} 1^{\prime}\right)$ is $0.091 \mathrm{~V}$. These observations indicate a fairly reversible process. ${ }^{72}$ These observations reveal that the redox process is due to surface confined viologen moieties. The coverage was estimated to be $(3.5 \pm 0.3) \times$ $10^{-10} \mathrm{~mol} \mathrm{~cm}{ }^{-2}$, which corresponds to an area of $0.47 \mathrm{~nm}^{2}$ per molecule. This value is in good agreement with literature data reported for related systems. ${ }^{53,56-58,60}$ It is significantly smaller than the projected area of a planar oriented HS-6V6-H molecule ( $c f$. crystal structure data in refs. 73 and 74) but larger than the estimated cross section of an all-trans oriented alkanethiol in a densely packed self-assembled monolayer. ${ }^{75}$ This qualitative discussion points to a tilted orientation of the viologen moiety with respect to the surface normal as suggested by Sagara. ${ }^{59}$ Attempts to estimate a 
rate constant from the variation of the peak separation on the scan rate andibasddicon Corlimenoamperometric experiments ${ }^{72}$ with microelectrodes indicated that $k_{\mathrm{o}}$ (at $\left.E_{\mathrm{P} 1 / \mathrm{P1}^{\prime}}\right)>150 \mathrm{~s}^{-1}$. ${ }^{76}$ The second pair of peaks $\mathrm{P} 2(E=-0.920 \mathrm{~V})$ and $\mathrm{P} 2^{\prime}(E=-0.900 \mathrm{~V})$ is assigned to $\mathrm{V}^{+} \cdot \leftrightarrow \mathrm{V}^{51,52}$ The peak-to-peak separation is constant up to $1 \mathrm{~V} \mathrm{~s}^{-1}$ and the peak height scales linearly with the scan rate. The peak FWHM of $\mathrm{P} 2$ and $\mathrm{P} 2^{\prime}$ are estimated to be $0.110 \mathrm{~V}$ and $0.132 \mathrm{~V}$, respectively. The broadening indicates quasi-reversibility and higher disorder within the adlayer. The latter might be attributed partially to the superposition with the process at $\mathrm{P} 3 / \mathrm{P}^{\prime}$. The narrow peak $\mathrm{P} 3$ at $E=-1.080 \mathrm{~V}, \mathrm{FWHM}=0.023 \mathrm{~V}$, is assigned to the reductive desorption of the redox-active adlayer. Current integration, after correction of the double layer contribution, yields a desorption charge of approximately $(160 \pm 10) \mu \mathrm{C} \mathrm{cm}^{-2}$. This value is considerably larger than $70 \mu \mathrm{C} \mathrm{cm}$ typically reported for alkanethiols, ${ }^{71}$ and indicates contributions of parallel processes such as bromide (perchlorate) desorption and cation (potassium) re-adsorption. The occurrence of the corresponding anodic peak $\mathrm{P}^{\prime}$ at $E=-0.950 \mathrm{~V}$ points to a partial oxidative re-adsorption of HS$6 \mathrm{~V} 6-\mathrm{H}$ on $\mathrm{Au}(111)-(1 \times 1)$. Multiple cycling in $-1.150 \mathrm{~V}<E<-0.200 \mathrm{~V}$ results in rather distorted current-voltage characteristics and shall not be considered further.

Systematic studies on the modification of $\mathrm{Au}(111)-(1 \times 1)$ with HS-6V6-H revealed that a complete monolayer was obtained upon deposition from $1 \mathrm{mM}$ ethanolic solution and annealing at $70{ }^{\circ} \mathrm{C}$ at incubation times longer than $1 \mathrm{~h}$. No evidence for multilayer formation was obtained. Using lower concentrations of HS-6V6-H and shorter immersion times resulted, in a wide range of exposure times and after a subsequent thermal treatment in viologen-free ethanol, to rather stable coverages of the fully oxidized form of HS-6V6-H clustering around $(0.9 \pm 0.2) 10^{-10} \mathrm{~mol} \mathrm{~cm}^{-2}$. The redox-response of a film prepared according to protocol 2 ( $c f$. Section 2.3) is presented as a dotted line in Fig. 1A, for clarity only in a limited potential range.

$\mathrm{Au}(111)-(1 \times 1)$ electrodes modified with the dithiol HS-6V6-SH, bromide salt, according to protocol 3 (Fig. 1B) gave results similar to those just described for HS-6V6-H: The double layer capacitance of a freshly prepared film amounts to $10 \mu \mathrm{F} \mathrm{cm} \mathrm{cm}^{-2}$. The first pair of redox peaks is rather reversible with $\mathrm{P} 1(E=-0.438 \mathrm{~V}, \mathrm{FWHM}=0.125 \mathrm{~V})$ and $\mathrm{P}^{\prime}(E=-0.419 \mathrm{~V}, \mathrm{FWHM}=0.095 \mathrm{~V})$, and scales linearly with the scan rate. Careful exclusion of oxygen during the assembly and characterization steps resulted in a rather symmetric peak shape. The peak current scales linearly with scan rate. The monolayer coverage is estimated to be $(4.5 \pm 0.5) \times 10^{-10} \mathrm{~mol} \mathrm{~cm}^{-2}$, which corresponds to an area of $0.37 \mathrm{~nm}^{2}$ per molecule. The second pair of peaks is less reversible, $\mathrm{P} 2(E=$ $-0.970 \mathrm{~V}, \mathrm{FWHM}=0.136 \mathrm{~V}), \mathrm{P}^{\prime}(E=-0.840 \mathrm{~V})$, and one often observes a splitting of $\mathrm{P} 2^{\prime}$. The reductive desorption occurs at $\mathrm{P} 3(E=-1.210 \mathrm{~V}, \mathrm{FWHM}=0.028 \mathrm{~V})$, and consumes a charge of $140 \mu \mathrm{C} \mathrm{cm}^{-2}$. Partial oxidative re-adsorption is observed at $\mathrm{P}^{\prime}(E=-0.935 \mathrm{~V})$. The larger values of the FWHM of both oxidation/reduction peaks attributed to the viologen moiety in HS-6V6-SH indicate repulsive interaction and a higher disorder within the dithiol viologen adlayer as compared to the monothiol film. The higher value of the interfacial capacitance and the more negative reductive desorption peak P3 may represent a more hydrophilic electrolytelsulfur end group interface than in the case of the $\mathrm{CH}_{3}$-terminated monothiol. The latter trend was also observed in a comparative study of alkane mono- and dithiols on Au(111). ${ }^{77}$ However, one important difference between HS-6V6-SH and HS-6V6-H needs to be mentioned: The presence of oxygen during the assembly process resulted in a distorted redox-response with indications of multi-layer formation. The strict absence of oxygen allowed us to prepare, for a wide range of conditions, adlayers with stable HS-6V6-SH coverages of $\Gamma=(1.1 \pm 0.2) \times 10^{-10} \mathrm{~mol} \mathrm{~cm}^{-2}$ according to protocol 2 (Section 2.3).

Comparing the potentials of the characteristic redox-processes $\mathrm{V}^{2+} \leftrightarrow \mathrm{V}^{+} \cdot$ and $\mathrm{V}^{+} \cdot \leftrightarrow \mathrm{V}$ for HS-6V6-SH and HS-6V6-H under the present experimental conditions with literature data ${ }^{51-54,56-60,63}$ indicates that co-adsorbed bromide ions are partially replaced by perchlorate ions, which are in excess in the supporting electrolyte chosen in this study.

\subsection{Cyclic voltammetry with gold nano-electrodes}

The knowledge gained in the preparation and characterization of HS-6V6-H and HS-6V6-SH modified $\mathrm{Au}(111)-(1 \times 1)$ macro-electrodes was applied to immobilize the viologen derivatives on electrochemically etched gold nano-electrodes (Fig. 2), which were coated with an insulating layer of polyethylene except at the apex. The electrode area exposed to the electrolyte after coating was 


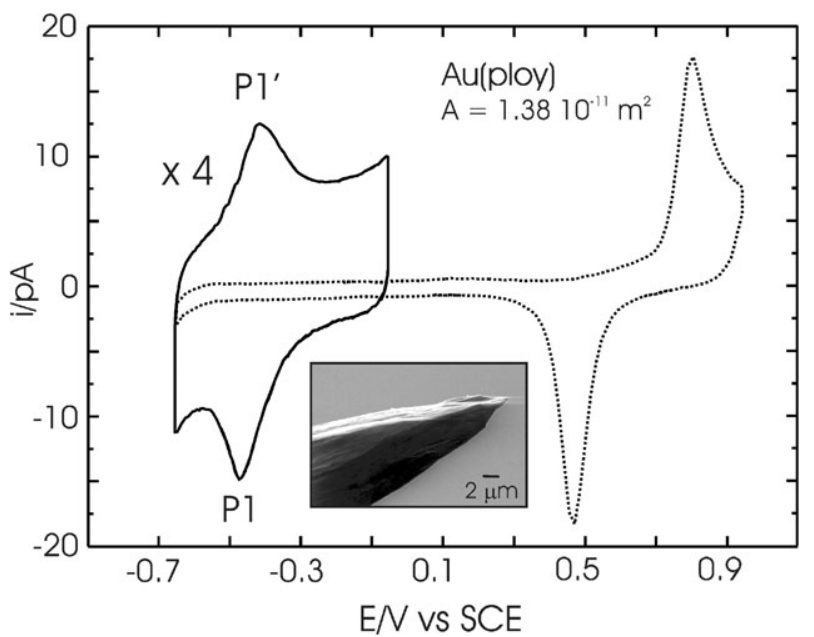

View Article Online

Fig. 2 Cyclic voltammograms of a bare (dotted line) and a HS-6V6-H-modified (solid line) gold nano-electrode in $0.05 \mathrm{M} \mathrm{KClO}_{4}$, adjusted with phosphate buffer to $\mathrm{pH} \mathrm{7,} \mathrm{sweep} \mathrm{rate} 50 \mathrm{mV} \mathrm{s}$. The inset shows a SEM micrograph of a freshly etched, uncoated electrode.

estimated from calibration experiments with polycrystalline macro-electrodes of known geometry based on the charges consumed during the gold surface oxidation and reduction. The dotted trace in Fig. 2 shows the voltammograms of a typical gold nano-electrode in $0.05 \mathrm{M} \mathrm{KClO}_{4}$, adjusted with phosphate buffer to $\mathrm{pH}$ 7. The effective free electrode area was estimated to $1.4 \times 10^{-11} \mathrm{~m}^{2}$. The SEM image of the uncoated tip is displayed as an inset in Fig. 2. Immobilization of the redox-active viologen was carried out by exposing the very end of the coated gold STM tip to a $1 \mathrm{mM}$ ethanolic solution of HS-6V6-H or HS-6V6-SH in a controlled argon atmosphere for $1 \mathrm{~h}$. Subsequently, the tip was carefully rinsed with pure ethanol, dried in a stream of argon, and immersed in a custommade electrochemical cell to access its activity. The solid trace in Fig. 2 reveals, as an example, a well-behaved redox-response $\mathrm{V}^{2+} \leftrightarrow \mathrm{V}^{+}$of surface immobilized HS-6V6-H. The oxidation and reduction peaks were found at $E=-0.428 \mathrm{~V}$ and $E=-0.450 \mathrm{~V}$. The peak height scales rather linearly with scan rate. The amount of immobilized viologen was derived from the charges of the oxidation and reduction peaks, after background subtraction and assuming a one-electron redoxprocess, to $5 \times 10^{-12} \mathrm{C}$. Referring to the free electrode area one obtains as an estimation of the surface coverage $3.7 \times 10^{-10} \mathrm{~mol} \mathrm{~cm}{ }^{-2}$. Similar results were also obtained with HS-6V6-SH. These observations are in very good agreement with the results reported for $\mathrm{Au}(111)-(1 \times 1)$ macroelectrodes indicating that the viologens retain their redox-functionality. Such modified nanoelectrodes are suitable candidates to explore tunneling characteristics of redox-molecule modified nanogaps by current-(bias) voltage spectroscopy employing an electrochemical STM configuration.

\subsection{SEIRAS experiments on self-assembly and redox-functionality}

The assembly of HS-6V6-H and HS-6V6-SH on quasi-single crystalline Au(111-25 nm) film electrodes was first monitored in ethanolic solution without potential control. We ensured that the formation and characterization of the adlayers was strictly carried out in the absence of oxygen. Exposure to $50 \mu \mathrm{M}$ solution of the respective viologen derivatives for $2 \mathrm{~min}$, subsequent rinsing with pure ethanol to remove second-layer adsorbates, and drying of the adlayer in a stream of argon, all within the same spectroelectrochemical cell, ${ }^{49}$ resulted only in rather weak bands in the $\mathrm{C}-\mathrm{H}$ stretching region. No bands related to in-plane vibrations of the bipyridinium ring were observed. Application of the surface selection rules of SEIRAS indicates that the above preparation yields viologen adlayers, where the aromatic ring appears to be oriented close to parallel to the gold surface. Longer assembly times in $1 \mathrm{mM}$ ethanolic viologen containing solution gave high coverage monolayers with prominent vibration modes in the $3200-2800 \mathrm{~cm}^{-1}$ and $1750-1100 \mathrm{~cm}^{-1}$ regions 

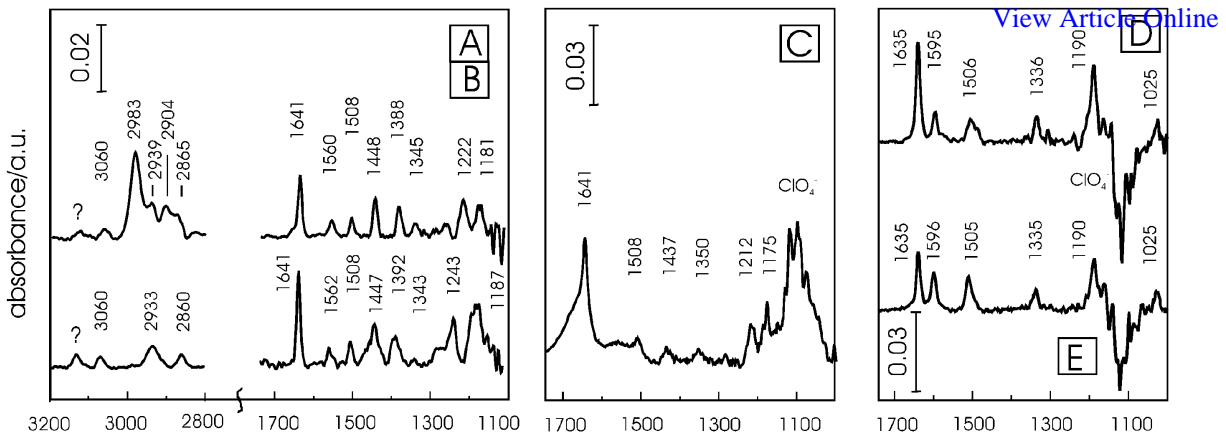

wavenumber $\mathrm{v} / \mathrm{cm}^{-1}$

Fig. 3 SEIRAS spectra for both viologen derivatives on $\mathrm{Au}(111-25 \mathrm{~nm})$ electrodes. Fig. 3A and Fig. 3B represent the spectral regions 3200 to $2800 \mathrm{~cm}^{-1}$ and 1750 to $1100 \mathrm{~cm}^{-1}$ for the assembly of high coverage monolayers from $1 \mathrm{mM}$ ethanolic solutions of HS-6V6-H (A) and HS-6V6-SH (B) without potential control. The assembly time was $2 \mathrm{~h}$. The plotted absorption spectra refer to the "dry" organic monolayers obtained after solution exchange, careful rinsing with bare ethanol and subsequent evaporation of the solvent in a gentle stream of argon. The reference spectrum was measured for the bare gold electrode in contact with dried argon. Fig. 3C represents the SEIRAS spectrum of a monolayer of the dication form of HS-6V6-H $\left(\mathrm{V}^{2+}\right)$ recorded at $E=$ $-0.200 \mathrm{~V}$ in $0.05 \mathrm{M} \mathrm{KClO}_{4}, \mathrm{pH}$ adjusted to 7 . The reference spectrum was obtained in the absence of viologen in $0.05 \mathrm{M} \mathrm{KClO}_{4}, \mathrm{pH}=7$, at $E=-0.200 \mathrm{~V}$. The formation of the self-assembled adlayer was carried out from $1 \mathrm{mM}$ ethanolic HS-6V6-H for $2 \mathrm{~h}$. Fig. 3D and 3E represent SEIRAS spectra of the radical cations $\mathrm{V}^{+}{ }^{\circ}$ of HS-6V6-H (D) and HS-6V6-SH (E) obtained after a potential scan from $E_{\mathrm{i}}=-0.200 \mathrm{~V}$ to $E_{\mathrm{f}}=-0.600 \mathrm{~V}$. The reference spectra were measured at $E_{\mathrm{i}}=-0.200 \mathrm{~V}$.

(Fig. 3A and 3B). The reference spectra were acquired for the bare gold surface. The data for HS6V6-H shall be described in detail. Differences of the HS-6V6-SH adlayer will be addressed comparatively. The bands in $3200-2800 \mathrm{~cm}^{-1}$ could be assigned to $\mathrm{C}-\mathrm{H}$ stretching vibrations of the aromatic ring $\left(v_{\mathrm{CH}}\right.$ at $\left.3060 \mathrm{~cm}^{-1}\right)$, the $\mathrm{CH}_{3}$-asymmetric $\left(v_{\mathrm{a}}\left(\mathrm{CH}_{3}\right)\right.$ at $\left.2983 \mathrm{~cm}^{-1}\right)$ and symmetric $\left(v_{\mathrm{s}}\left(\mathrm{CH}_{3}\right)\right.$ at $\left.2904 \mathrm{~cm}^{-1}\right)$, respectively, the $\mathrm{CH}_{2}$-asymmetric $\left(v_{\mathrm{a}}\left(\mathrm{CH}_{2}\right)\right.$ at $\left.2939 \mathrm{~cm}^{-1}\right)$ and the symmetric $\left(v_{\mathrm{s}}\left(\mathrm{CH}_{2}\right)\right.$ at $\left.2865 \mathrm{~cm}^{-1}\right)$ stretching modes. ${ }^{78-80}$ The vibration modes $v_{\mathrm{a}}\left(\mathrm{CH}_{3}\right)$ and $v_{\mathrm{s}}\left(\mathrm{CH}_{3}\right)$ are absent for the HS-6V6-SH adlayer (Fig. 3B). The observed spectroscopic bands below $1750 \mathrm{~cm}^{-1}$ represent vibration modes of the bipyridinium ring having dipole moment components either parallel to the longer molecular axis, as defined by the $4,4^{\prime}-N, N^{\prime}$ - direction $\left(B_{2 \mathrm{u}}\right.$ in $D_{2 \mathrm{~h}}$ symmetry; $1641,1508,1222$ and $1181 \mathrm{~cm}^{-1}$ with the latter dominated by the $N-\left(\mathrm{CH}_{2}\right)_{n}$ - $\mathrm{R}$ stretching vibration), or perpendicular to this axis ( $B_{3 \mathrm{u}}$ modes in $D_{2 \mathrm{~h}}$ symmetry; $\left.1560,1448,1345 \mathrm{~cm}^{-1}\right){ }^{78-83}$

The positions of the methylene $\mathrm{C}-\mathrm{H}$-stretching modes provide a measure of the intermolecular environment of the alkyl chains in the molecular assembly. The peak frequencies of $v_{\mathrm{s}}\left(\mathrm{CH}_{2}\right)(2865$ vs. $\left.2850 \mathrm{~cm}^{-1}\right)$ and $v_{\mathrm{a}}\left(\mathrm{CH}_{2}\right)\left(2939\right.$ vs. $\left.2920 \mathrm{~cm}^{-1}\right)$ in the liquid state are typically higher than those for a highly ordered crystalline polymethylene chain. ${ }^{84}$ The applications of Porter's results to the present system leads to the conclusion that the methylene chain environment of the high coverage adlayers of HS-6V6-H and HS-6V6-SH are liquid-like, i.e., there exists a considerable degree of conformational disorder in the two-dimensional arrangement of the alkyl chains. The observation of the aromatic $\mathrm{C}-\mathrm{H}$ stretching mode at $3060 \mathrm{~cm}^{-1}$, together with the simultaneous occurrence of the $B_{2 \mathrm{u}}$ and $B_{3 \mathrm{u}}$ in-plane bipyridinium ring vibrations, indicate a tilted orientation of both, the long and the short axis of the coplanar viologen moiety ${ }^{73,78-80}$ with respect to the surface normal. Support for this conclusion is provided by recent results with related systems. Sagara et al. ${ }^{57}$ concluded, using electro-reflectance spectroscopy that the long axis of the viologen group in $N$-butyl- $N^{\prime}$-(4-thiobutyl)-4,4'-bipyridinium dihexaflourophosphate on Au(poly) exhibits an orientation angle of $70^{\circ}$ with respect to the surface normal. Based on a combined infrared and near edge X-ray absorption fine structure spectroscopy study Rong et al. ${ }^{85}$ reported tilting and leaning angles of $45^{\circ}$ and $70^{\circ}$, respectively, for the aromatic moiety in monolayers of $\omega$-( $4^{\prime}$-methyl-biphenyl-4-yl)alkanethiols with an even number of methylene spacer groups on $\mathrm{Au}(111)$. 
The above knowledge on the assembly of HS-6V6-H and HS-6V6-SH on gold firom thimantines applied to explore the adlayer properties under electrochemical conditions in $0.05 \mathrm{M} \mathrm{KClO}_{4}$ with a $\mathrm{pH}$ adjusted to either 7 or (in some cases) to 10 . Because of stability reasons of the adlayer we restricted the spectroelectrochemical studies to the potential region $-0.700 \mathrm{~V} \leq E \leq-0.200 \mathrm{~V}$, e.g., to $\mathrm{V}^{2+} \leftrightarrow \mathrm{V}^{+} \cdot$. High coverage monolayers on $\mathrm{Au}(111,25 \mathrm{~nm})$ were prepared according to protocol 3 in paragraph 2.3. The in situ SEIRAS spectra were recorded with reference to the adsorbate free electrolyte at $-0.200 \mathrm{~V}$. While the spectral signature in the $\mathrm{C}-\mathrm{H}$ stretching region appears to be similar to the results in bare ethanol (Fig. 3A), we observed distinct changes in $1750-1000 \mathrm{~cm}^{-1}$. The vibrations with net dipole moment changes parallel to the molecular axis defined by the 4,4'$N, N^{\prime}$-direction $\left(B_{2 \mathrm{u}}, 1641,1508,1212,1175 \mathrm{~cm}^{-1}\right)$ appear with a higher intensity than the $B_{3 \mathrm{u}}$ modes $\left(1437,1350 \mathrm{~cm}^{-1}\right)$. This observation points to a stronger alignment of the viologen moiety with respect to the surface normal. Furthermore, we notice that the bipyridinium ring vibration at 1641 $\mathrm{cm}^{-1}$ is superimposed with a rather broad $\mathrm{HOH}$ bending mode of co-adsorbed interfacial water, $\delta_{\mathrm{HOH}}$. This finding supports our previous hypothesis based on values of the interfacial capacitance. The prominent positive peak at $1100 \mathrm{~cm}^{-1}$ is attributed to the symmetric $\mathrm{Cl}-\mathrm{O}$ stretching mode of perchlorate ions incorporated into the HS-6V6-H respective HS-6V6-SH adlayer lattice. The presence of perchlorate ions increases the $2 \mathrm{D}$ order within the organic film as derived from peak shapes and intensities of the $B_{2 u}$ modes. The replacement of bromide or chloride ions in selfassembled monolayers of thiol-functionalized viologens by ions with a smaller dynamic hydration sphere, such as $\mathrm{ClO}_{4}{ }^{-}$and $\mathrm{PF}_{6}{ }^{-}$, was already suggested in voltammetry studies of Buttry ${ }^{53,54}$ and Sagara. ${ }^{58}$ The lower electron donor ability of $\mathrm{ClO}_{4}{ }^{-}$reduces the charge transfer between the viologen moiety and the co-adsorbed anion. ${ }^{74}$

Increasing the electrode potential from $E=-0.200 \mathrm{~V}$ to $E=-0.600 \mathrm{~V}$ triggers the one electron reduction of $\mathrm{V}^{2+} \leftrightarrow \mathrm{V}^{+}$. The simultaneously acquired SEIRAS difference spectra show the evolution of strong positive bands around 1635, 1595, 1506 (5), 1336 (5), 1190 and $1025 \mathrm{~cm}^{-1}$ for both viologen adlayers. The spectroscopic response is completely reversible, and agrees well with our voltammetry studies (paragraph 3.1.). The data acquired at $E=-0.600 \mathrm{~V}$ are plotted in Fig. 3D and Fig. 3E. The prominent bands at 1635 and $1190 \mathrm{~cm}^{-1}$ can be assigned to the asymmetric inplane vibrations of the monomer radical cation $\mathrm{V}^{+}$. The others are attributed to "activated" totally symmetric $\left(A_{\mathrm{g}}\right)$ ring vibrations of the radical cation dimer $\left(\mathrm{V}^{+} \bullet\right)_{2}$, in which plane-to-plane arranged molecules are linked by $\pi$-bonding. ${ }^{78-83}$ The IR spectrum of the electrochemically generated dimer species is interpreted by the vibronic coupling in a one-dimensional charge transfer complex. ${ }^{82}$ The negative band around $1100 \mathrm{~cm}^{-1}$ indicates the release of perchlorate ions upon the reduction $\mathrm{V}^{2+}$ $\leftrightarrow \mathrm{V}^{+} \cdot$. The radical cation monomer is stabilized in a hydrophobic environment ${ }^{80}$ and by electrostatic attraction to co-adsorbed $\mathrm{ClO}_{4}{ }^{-}$anions. The spectra of HS-6V6-H (Fig. 3D) and HS-6V6-SH (Fig. 3E) demonstrate that the stability of the radical cation monomer appears to be higher in the monothiol adlayer. The formation of the radical cation dimer for the dithiol adlayer is facilitated by stronger $\pi$-interactions between the radicals compared to Coulomb interactions between radical monomers and anions, ${ }^{82}$ as well as by the matching in the commensurability of the intra-assembly planes. $^{86}$

A comprehensive analysis of the steady state and time-resolved ATR-SEIRAS experiments on the self-assembly and electroreduction of HS-6V6-H and HS-6V6-SH on gold Au(111, $25 \mathrm{~nm})$ will be given elsewhere. ${ }^{87}$

\subsection{Interfacial structure of HS-6V6-H and HS-6V6-SH}

In situ STM was applied to monitor viologen adlayers assembled according to the preparation protocols (1) to (3). Exposure of an $\mathrm{Au}(111)-(1 \times 1)$ electrode to a $50 \mu \mathrm{M}$ ethanolic solution of HS6V6-H or HS-6V6-SH for $1 \mathrm{~min}$ generated a low coverage, liquid-like disordered adlayer (protocol 1). Modification according to protocol 2 ( 2 min contact with $50 \mu \mathrm{M}$ ethanolic viologen solution, 12 $\mathrm{h}$ annealing in pure ethanol at $70{ }^{\circ} \mathrm{C}$ ) resulted in highly ordered, but extremely fragile adlayers. Both HS-6V6-H (Fig. 4A and 4B) and HS-6V6-SH (Fig. 4D and 4E) assemble on Au(111)- $(1 \times 1)$ in $-0.70 \mathrm{~V} \leq E \leq-0.20 \mathrm{~V}$, i.e., the stability ranges of $\mathrm{V}^{2+}$ and $\mathrm{V}^{+} \cdot\left(\mathrm{V}^{+} \cdot\right)_{2}$, in regular arrays of parallel stripes, which cover the entire substrate surface. Typical results for $E=-0.20 \mathrm{~V}$ are summarized in Fig. 4. The stripes are not uniform. Bright and dark contrast patterns alternate. Individual domains extend 10 to $50 \mathrm{~nm}$. Neighboring domains with sharp boundaries are mutually 


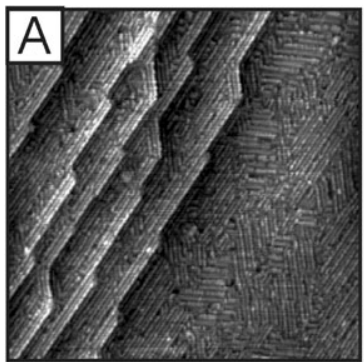

$100 \mathrm{~nm}$

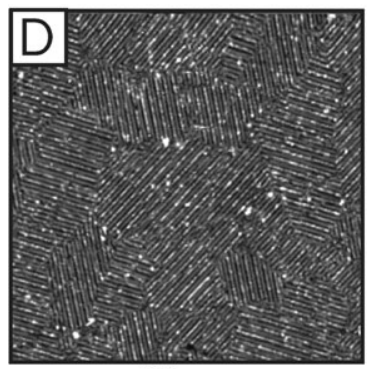

$100 \mathrm{~nm}$

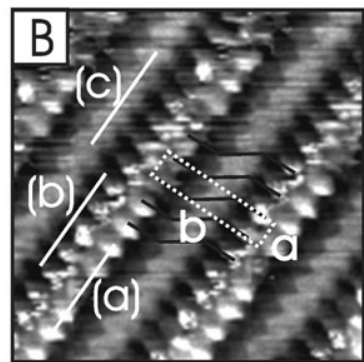

$7 \mathrm{~nm}$

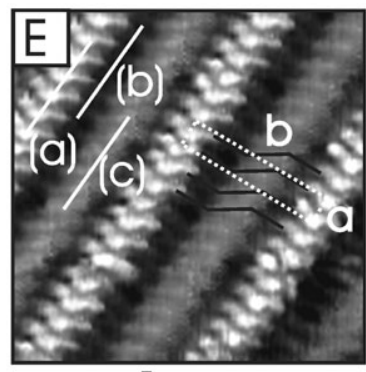

$7 \mathrm{~nm}$

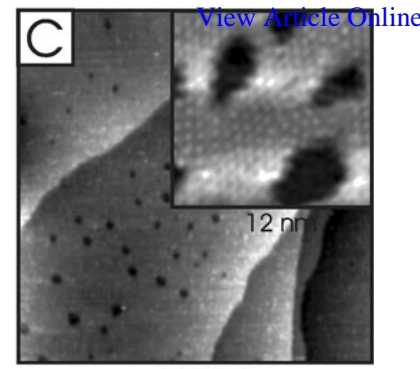

$100 \mathrm{~nm}$

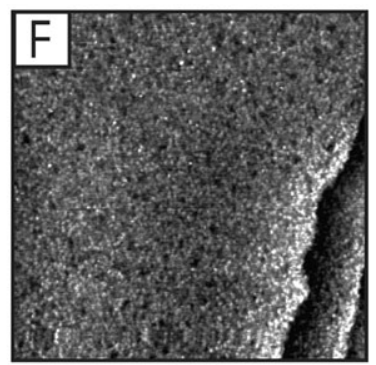

$100 \mathrm{~nm}$

Fig. 4 In situ STM images of HS-6V6-H (Fig. A, B and C) and of HS-6V6-SH (Fig. D, E, F) on Au(111)-(1 × 1) in $0.05 \mathrm{M} \mathrm{KClO}_{4}, \mathrm{pH}=7$, recorded at $E_{\mathrm{S}}=-0.200 \mathrm{~V}$. The setpoint current was typically between 50 and 100 $\mathrm{pA}$. The images represent the low-coverage "striped" adlayers (A, B, D and E) assembled according to protocol 2, and the high coverage monolayers formed according to protocol 3 (Fig. 4C and F) in paragraph 2.3 (see text for details). The high resolution images show distinct row patterns, which are indicated by the traces (a), (b) and (c). The typical zig-zag motif of the aligned segments is drawn as a sequence of black solid lines. The dotted rectangles illustrate an adlayer repeat pattern with $a=2.6 \mathrm{~nm}$ and $b=0.5 \mathrm{~nm}$. Table 1 summarizes characteristic parameters of the viologen adlayers on $\mathrm{Au}(111)-(1 \times 1)$.

rotated by multiples of $120^{\circ}$ indicating registry of the adlayer with respect to the hexagonal substrate surface. This hypothesis is supported by the faceting of steps (Fig. 4A). Steps are aligned with characteristic angles of $120^{\circ}$. Step edges are decorated by parallel molecular rows. Occasionally one observes monatomically high gold and/or vacancy islands at domain boundaries. High resolution experiments such as shown in Fig. 4B and 4E reveal more details of the molecular adlayers. Rows composed of two (HS-6V6-H) or a more complex pattern (HS-6V6-SH) of bright dots (trace a) are separated by parallel rows of dark grey (trace b) respective light grey (trace c) ellipsoidal-like segments. Their axes exhibit characteristic tilt angles with respect to the main row direction, which were estimated to be $60 \pm 5^{\circ}$ (light grey rows) and $90 \pm 10^{\circ}$ (dark grey rows). The resulting, nearly equilateral zig-zag motif is indicated in both high resolution images. Parallel rows of the same type are separated by $(2.6 \pm 0.3) \mathrm{nm}$ and $(2.7 \pm 0.3) \mathrm{nm}$ for HS-6V6-H and HS-6V6-SH, respectively. Cross section profiles reveal a common periodicity of $(0.50 \pm 0.05) \mathrm{nm}$ between individual features of each type of row, but distinct differences in the apparent corrugation height. Fig. 5 illustrates the corresponding results for HS-6V6-H. We estimated 0.14 to $0.19 \mathrm{~nm}$ (trace a), 0.03 to $0.06 \mathrm{~nm}$ (trace b) and 0.05 to $0.12 \mathrm{~nm}$ (trace c). Similar data were obtained for HS-6V6-SH. The above results suggest a commensurate repeat motif with $\mathrm{a}=0.50 \pm 0.05 \mathrm{~nm}\left(=\sqrt{ } 3 a_{\mathrm{Au}}\right)$ parallel and $\mathrm{b}=2.6 \pm 0.3 \mathrm{~nm}\left(\sim 9 a_{\mathrm{Au}}\right)$ perpendicular to the molecular rows.

In an attempt to derive structural models of the two adlayers we notice that the values of the apparent corrugation heights are similar to data reported for alkanethiols ${ }^{88}$ aligned with their $\mathrm{C}-\mathrm{C}-$ $\mathrm{C}$ backbone parallel, and for aromatic molecules ${ }^{89}$ oriented with the $\pi$-system of the phenyl ring planar or slightly tilted to the $\mathrm{Au}(111)$ surface. This arrangement appears to be applicable for the two viologen adlayers prepared according to protocol 2. The conclusion is also supported by our 


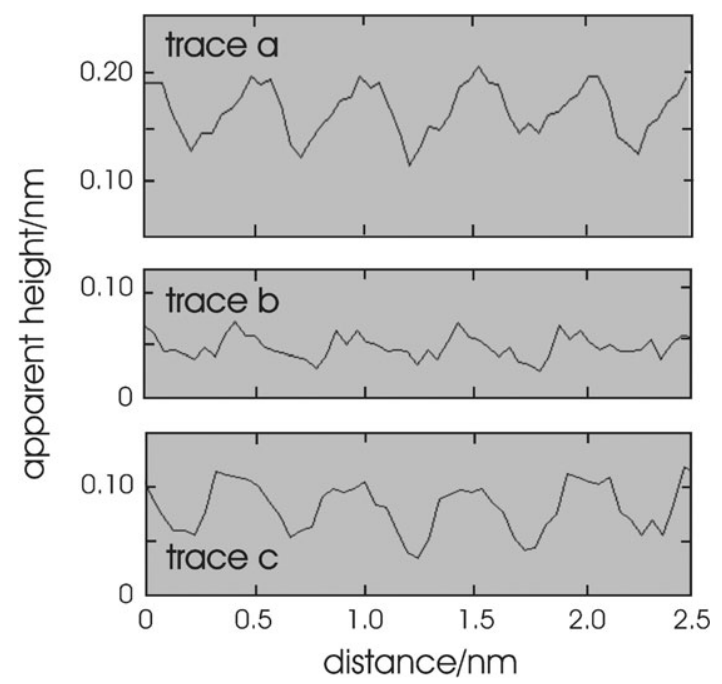

View Article Online

Fig. 5 Typical cross section profiles along the traces drawn in Fig. 4B for the striped adlayer of HS-6V6-H on $\mathrm{Au}(111)-(1 \times 1)$ in $0.05 \mathrm{M} \mathrm{KClO}_{4}$ at $E=-0.200 \mathrm{~V}$.

SEIRAS results (paragraph 3.3). Striped adlayers of molecules aligned parallel to the substrate surface have been described for low coverage phases of alkanethiols, ${ }^{77,88,90}$ alkanedithiols ${ }^{77,91,92}$ and several $n$-phenyl-alkanethiols on $\mathrm{Au}(111) \cdot{ }^{93-95} \mathrm{An}$ enhancement of the tunneling current was observed in the vicinity of the sulfur group, often apparent as bright spots or slightly elongated features, ${ }^{90-92}$ while the alkyl chains are observed as thin bands of rather dark contrast. ${ }^{90,91}$ Aromatic groups, such as phenyl-, biphenyl- or bipyridine-units were also reported to enhance the tunneling contrast. ${ }^{89,96-98}$ In applying this knowledge to the present case we notice that the width of the dark rows (trace b) increases with the length of the alkyl chains when replacing the hexyl groups by octyl $\left(\mathrm{C}_{8}\right)$ or decyl $\left(\mathrm{C}_{10}\right)$ in HS-6V6-H respective HS-6V6-SH, while all other features in the bright and light grey rows do not change. ${ }^{87}$ The dimensions of the corresponding repeat motifs, which all indicate commensurability to the underlying $\mathrm{Au}(111)$ lattice, are compiled in Table 1 . The values of $b$ increase approximately $0.5 \mathrm{~nm}$ per $\left(\mathrm{CH}_{2}\right)_{2}$-unit, which exactly scales with twice the distance between neighboring $\mathrm{CH}_{2}$-groups. ${ }^{99}$ In consequence, we assign the dark rows to the positions of the alkyl chains in a fully extended all-trans conformation with the molecular axis parallel to the surface plane. Although we could not resolve the gold lattice separately in these experiments, we assume, based on adsorption studies of alkanes on $\mathrm{Au}(111),{ }^{99}$ that the $\mathrm{C}-\mathrm{C}-\mathrm{C}$ axis aligns with the $\left[\begin{array}{lll}1 & \overline{1} & 0\end{array}\right]$ direction of the hexagonal substrate. The light grey lines in Fig. $4 \mathrm{~B}$ and $4 \mathrm{E}$ exhibit an internal structure, which resembles the arrangement of planar or extended stacking rows of bipyridine units in self-assembled monolayers. ${ }^{97,98}$ In analogy to these reports we attribute the light grey rows in the high resolution STM images tentatively to the positions of the viologen moieties. The intermolecular distance between neighboring units, $0.43 \mathrm{~nm}\left(=\sqrt{ } 3 a_{\mathrm{Au}} \sin 60^{\circ}\right)$ is too small to allow a plane-parallel arrangement to the substrate, but also too large for a perfect stacking structure. The latter is not expected to be stable due to electrostatic repulsion between $4,4^{\prime}$ bipyrdinium dications. Based on crystallographic studies with co-crystallized $\mathrm{ClO}_{4}^{-}$ions ${ }^{74}$ we propose a slightly tilted and twisted conformation of the two rings. The more open motif could also accommodate the co-adsorbed $\mathrm{ClO}_{4}{ }^{-}$ions. The exact arrangement could not be resolved in more detail. Despite the limited knowledge, we can assume a sufficiently open arrangement of the viologen units, which, most probably, is stabilized by co-adsorbed $\mathrm{ClO}_{4}^{-}$( $c f$. paragraph 3.3). Finally, we assigned the bright rows to the positions of the terminal sulfur functionalities. The suggested structural models of HS-6V6-H and HS-6V6-SH on $\mathrm{Au}(111) / 0.05 \mathrm{M} \mathrm{KClO}_{4}$ are drawn in Fig. 6. We assume an antiparallel head-to-tail $-\mathrm{S}_{\mathrm{CH}} \mathrm{CH}_{3}-$ configuration for the monothiol and a headto-head-SIS-configuration for the dithiol. The corresponding unit cells appear to be multiples of the characteristic repeat patterns (Fig. 4). They contain four (HS-6V6-H) respective two (HS-6V6- 


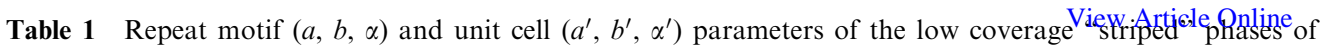
viologen mono- and dithiols on $\mathrm{Au}(111)-(1 \times 1)$

\begin{tabular}{llllllll}
\hline Monothiol & $\begin{array}{l}\text { Repeat } \\
a / \mathrm{nm}\end{array}$ & $\begin{array}{l}\text { Motif } \\
b / \mathrm{nm}\end{array}$ & $\alpha /{ }^{\circ}$ & $\begin{array}{l}\text { Unit } \\
a^{\prime} / \mathrm{nm}\end{array}$ & $\begin{array}{l}\text { Cell } \\
b^{\prime} / \mathrm{nm}\end{array}$ & $\alpha^{\prime} /{ }^{\circ}$ & $\Gamma / 10^{-10} \mathrm{~mol} \mathrm{~cm}^{-2}$ \\
\hline HS-6V6-H & $2.6 \pm 0.3$ & $0.50 \pm 0.05$ & $90 \pm 10$ & 5.35 & 1 & 87 & $1.24(1.10 \pm 0.20)^{b}$ \\
HS-8V8-H $^{a}$ & $3.15 \pm 0.20$ & $0.48 \pm 0.05$ & $89 \pm 8$ & 6.50 & 1 & 87.5 & 1.02 \\
HS-10V10H $^{a}$ & $3.8 \pm 0.3$ & $0.50 \pm 0.06$ & $90 \pm 7$ & 7.65 & 1 & 88 & 0.87
\end{tabular}

Dithiol

\begin{tabular}{llllllll}
\hline HS-6V6-SH & $2.7 \pm 0.3$ & $0.50 \pm 0.03$ & $90 \pm 8$ & 5.35 & 0.5 & 87 & $1.24(1.05 \pm 0.30)^{b}$ \\
HS-8V8-SH $^{a}$ & $3.2 \pm 0.3$ & $0.50 \pm 0.05$ & $88 \pm 7$ & 6.50 & 0.5 & 87.5 & 1.02 \\
HS-10V10-SH & $3.7 \pm 0.5$ & $0.50 \pm 0.05$ & $90 \pm 10$ & 7.65 & 0.5 & 88 & 0.87
\end{tabular}

${ }^{a} c f$. Ref. 87; ${ }^{b}$ Coverages estimated from electrochemical experiments such as plotted in Fig. 1.

$\mathrm{SH})$ molecules. The estimated coverage corresponds to the values of the voltammetry experiments (Table 1). The STM experiments give slightly higher values because the molecular disorder existing at defects and domain boundaries is not accounted for in the calculations.

When changing the electrode potential to $-0.600 \mathrm{~V}$, i.e., the radical cation $\mathrm{V}^{+} \cdot\left(\mathrm{V}^{+} \bullet\right)_{2}$ is present, we observed an increasing instability of the ordered molecular adlayers. While the main structural pattern is not changing, the light grey rows, previously attributed to the positions of the viologen moieties, could be easily distorted. This observation indicates a lower stability of the twodimensional adlayer pattern.

We also report on the high coverage adlayers of HS-6V6-H and HS-6V6-SH formed according to protocol 3. We obtained monolayers for both systems. The monothiol forms an expanded hexagonal adlayer, which covers the entire substrate surface (Fig. 4C). The structure resembles a $(\sqrt{ } 7 \times \sqrt{ } 7)$ motif. The resulting coverage amounts to $3.3 \times 10^{-10} \mathrm{~mol} \mathrm{~cm}{ }^{-2}$, which is in good agreement to the voltammetry data. The adlayer is composed of many, atomically deep vacancy islands with a typical size of 2 to $5 \mathrm{~nm}$ in diameter. The defects distort the molecular structure considerably. The open structure is expected due to the bulky size of the tilted viologen moiety ( $c f$. paragraph 3.3), which prevents a $(\sqrt{3} \times \sqrt{ } 3)$ arrangement. The latter is often observed for high coverage adlayers of thiols on $\mathrm{Au}(111){ }^{88,90,95}$

No ordered structure was found for the high coverage phase of upright HS-6V6-SH molecules on $\mathrm{Au}(111)-(1 \times 1)(\mathrm{Fig} .4 \mathrm{~F})$. The difference to the monothiols nicely illustrates the structuredetermining role of the alkyl chain above the aromatic group as already pointed out by Ulman et al. ${ }^{100}$ We did not observe any evidence of multilayer adsorption. However, the latter was detected if the experiment was carried out in the presence of oxygen.

\subsection{Addressing of local redox-activity}

Employing an in situ STM configuration (four electrode arrangement) we recorded current-distance and current-voltage characteristics ( $c f$. paragraph 2.5) to explore electron transport and tunneling properties of single and/or small assemblies of redox-active viologen derivatives immobilized on well-defined gold electrodes. Specifically, we aim to address the fundamental question how does the molecular conductivity change by tuning the redox state of the molecule (the "electrochemical gate effect").

Symmetric molecular junctions $\mathbf{A u ( T ) | H S - 6 V 6 - S H} \mid \mathbf{A u}(\mathbf{S})$. The first approach involves the repeated formation and breaking of molecular junctions between a polyethylene coated gold tip and a $\mathrm{Au}(111)-(1 \times 1)$ substrate electrode modified with HS-6V6-SH. The dithiol is composed of two SHanchor groups, which could bridge the tip and the substrate electrodes and form molecular junctions. We have chosen a low-coverage, liquid-like phase to prevent two-dimensional long- 


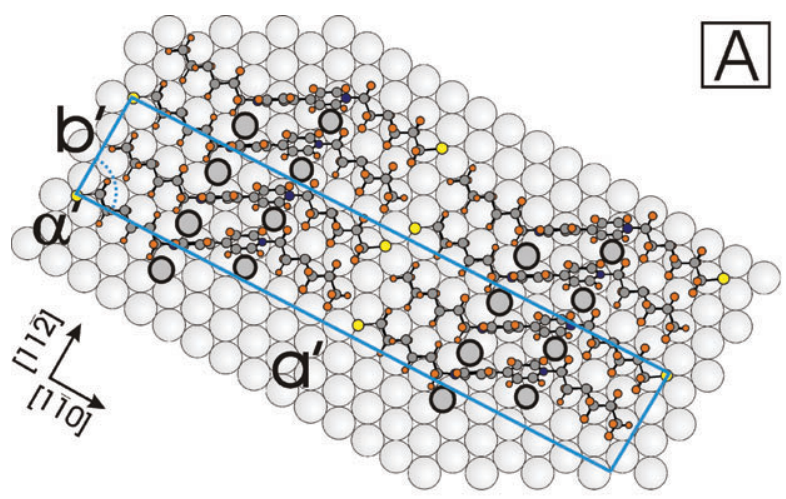

Fig. 6 Proposed packing models of the ordered striped phases of HS-6V6-H (A) and HS-6V6-SH (B) on $\mathrm{Au}(111)-(1 \times 1)$. Co-adsorbed perchlorate ions are drawn as open circles, and they are tentatively located next to the ring nitrogen atoms. Sulfur atoms are represented as yellow circles. Their suggested positions are three-fold hollow sites of the substrate lattice. The suggested unit cells, given by $\left(\begin{array}{cc}18 & 1 \\ -2 & 4\end{array}\right)$ (HS-6V6-H) and $\left(\begin{array}{cc}18 & 1 \\ -1 & 2\end{array}\right)$ (HS$6 \mathrm{~V} 6-\mathrm{SH})$ in matrix notation with reference to the basic lattice vectors of the $\mathrm{Au}(111)$, are indicated by bold solid lines. The unit cell parameters $a^{\prime}, b^{\prime}$ and $\alpha^{\prime}$ are summarized in Table 1, together with the dimensions of the experimentally estimated repeat motifs $a, b$, and $\alpha$.

range order within the organic adlayer, and therefore to allow for different surface conformations of planar oriented HS-6V6-SH molecules. The study was carried out under potential control in $0.05 \mathrm{M}$ $\mathrm{KClO}_{4}, \mathrm{pH}$ adjusted to 7, in a chamber filled with argon to prevent exposure to oxygen. Attempts to create individual $\mathrm{Au}(\mathrm{T})|\mathrm{HS}-6 \mathrm{~V} 6-\mathrm{SH}| \mathrm{Au}(\mathrm{S})$ junctions are based on slight, but distinct modifications of two techniques recently introduced by $\mathrm{TaO}^{25}$ and Haiss. ${ }^{26}$ The experiment started under feedback control with a setpoint current of $100 \mathrm{pA}$ ensuring negligible interactions between the STM tip and the organic adlayer. The tip-sample distance was estimated, according to eqn. (1): ${ }^{50}$

$$
\left|i_{\mathrm{T}}\right|=G_{\mathrm{o}}\left|E_{\mathrm{T}}-E_{\mathrm{S}}\right| \exp \left(-\kappa \sqrt{\Phi} z_{\mathrm{o}}\right)
$$

with $G_{\mathrm{o}}=2 e_{\mathrm{o}}{ }^{2} / h$ as the conductance quantum, $\kappa=10.12 \mathrm{eV}^{-1 / 2} \mathrm{~nm}^{-1}$ and assuming a rectangular potential barrier with a typical height $\Phi=1.0 \mathrm{eV},{ }^{50}$ one obtains for $\left|E_{\mathrm{T}}-E_{\mathrm{S}}\right|=0.10 \mathrm{~V} z_{\mathrm{o}} \sim 1.11$ $\mathrm{nm}$. This value was considered as the upper limit of the approaching distance $\Delta z_{\mathrm{a}}$. Typically, we have chosen $\left(z_{\mathrm{o}}-\Delta z_{\mathrm{a}}\right)$ with $\Delta z_{\mathrm{a}}=1.0 \mathrm{~nm}$ to ensure sufficiently strong interaction between the uncoated very end of the gold tip and the disordered HS-6V6-SH adlayer, but also to prevent 

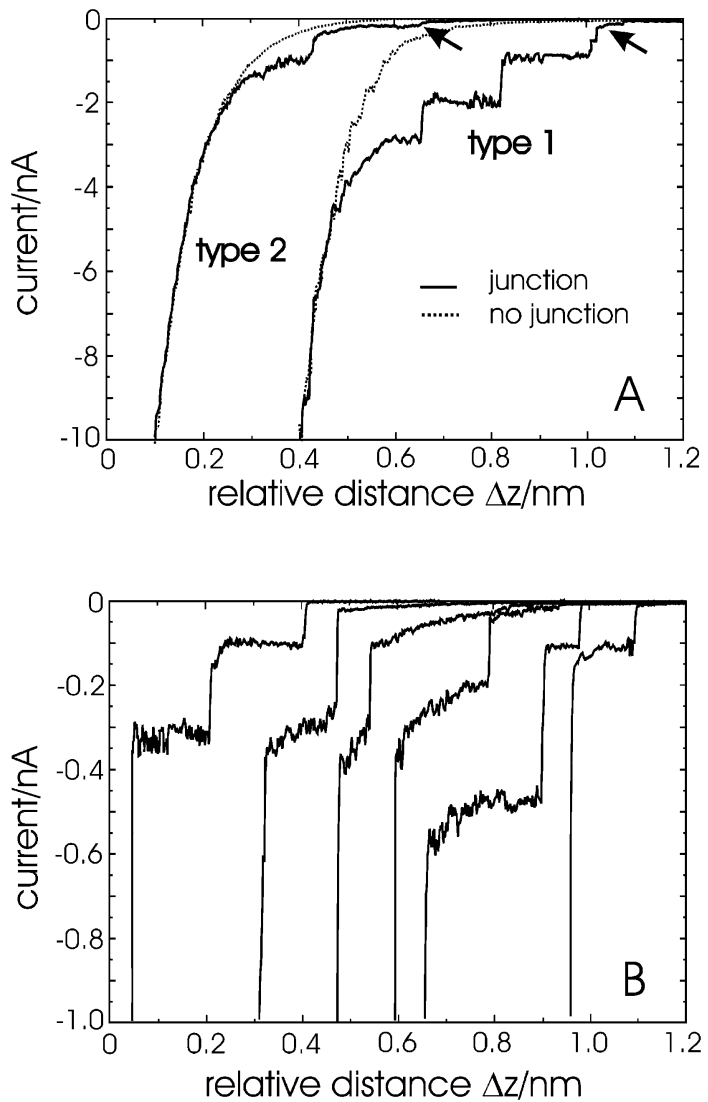

Fig. 7 Current-distance $(i-\Delta z)$ "pulling" curves recorded with a gold STM tip, coated with polyethylene except for the very end of the apex, in the presence of a disordered, low coverage HS-6V6-SH adlayer (protocol 1) on $\mathrm{Au}(111)-(1 \times 1)$ in $0.05 \mathrm{M} \mathrm{KClO}_{4}, \mathrm{pH}=7$, at $E_{\mathrm{S}}=-0.250 \mathrm{~V}$ and $E_{\mathrm{T}}=-0.150 \mathrm{~V}$. Individual traces were acquired according to a pre-defined loop described in detail in paragraph 3.5. The horizontal axes correspond to a relative distance scale. For clarity, individual traces are plotted with an offset. Fig. 7A illustrates representatives of two groups of $i-\Delta z$ curves characteristic for the breaking of molecular junctions Au(T)|HS-6V6$\mathrm{SH} \mid \mathrm{Au}(\mathrm{S})$. The solid traces are non-exponential with a series of plateaux, which are separated either by abrupt steps (type 1) or segments of gradually decreasing currents (type 2). The arrows indicate the positions of the lowest current plateaux. The dotted lines indicate $i-\Delta z$ curves in the absence of molecular junctions between gold tip and substrate. Fig. 7B shows the low current segments of a sequence of $i-\Delta z$ traces for the stretching of HS-6V6-SH junctions. The complete curves were recorded in $z_{\mathrm{O}}-1.0 \mathrm{~nm}<z<z_{\mathrm{O}}+2.0 \mathrm{~nm}$ and $0<i \leq 10 \mathrm{nA}$; $z_{\mathrm{o}}$ was defined by the initial conditions $i_{\mathrm{T}}=100 \mathrm{pA}$ and $\left(E_{\mathrm{T}}-E_{\mathrm{S}}\right)=0.100 \mathrm{~V}$.

mechanical contact to the gold surface. Individual traces were acquired according to the following loop: (1) feedback disabled at $z_{\mathrm{o}}$ (defined by $i_{\mathrm{o}}=100 \mathrm{pA}$ ), (2) approach with a maximum rate to $\left(z_{\mathrm{o}}-1.0 \mathrm{~nm}\right)$, (3) dwelling time of $100 \mathrm{~ms}$ at $\left(z_{\mathrm{o}}-1.0 \mathrm{~nm}\right)$ to form molecular junctions, (4) recording of the current $v s$. distance characteristics upon retraction from $\left(z_{\mathrm{o}}-1.0 \mathrm{~nm}\right)$ to $\left(z_{\mathrm{o}}+2.0\right.$ $\mathrm{nm}$ ) with a pulling rate of $6 \mathrm{~nm} \mathrm{~s}^{-1},(5)$ stabilizing of the tip at $z_{\mathrm{o}}$ and switching on the feedback with $i_{\mathrm{o}}=100 \mathrm{pA}$, (6) repetition of the cycle, and after every 10th trace inspection of the surface by in situ STM imaging. Fig. 7A shows the low current range of two characteristic stretching curves recorded at $E_{\mathrm{S}}=-0.250 \mathrm{~V}$ and $E_{\mathrm{T}}=-0.150 \mathrm{~V}$ in $0<i \leq 100 \mathrm{nA}$. The traces are non-exponential exhibiting a series of plateaux with a typical length of 0.2 to $0.3 \mathrm{~nm}$ which are separated by abrupt steps ("type 1 ") or by segments of a gradually decreasing current ("type 2"). The corresponding currents are rather low, and cannot be attributed to the well-known conductance quantization of a metal nanowire. ${ }^{25}$ Instead, we ascribe these conductance steps to the breaking of individual, respective multi-molecular (HS-6V6-SH) junctions previously formed between the gold STM tip and the 

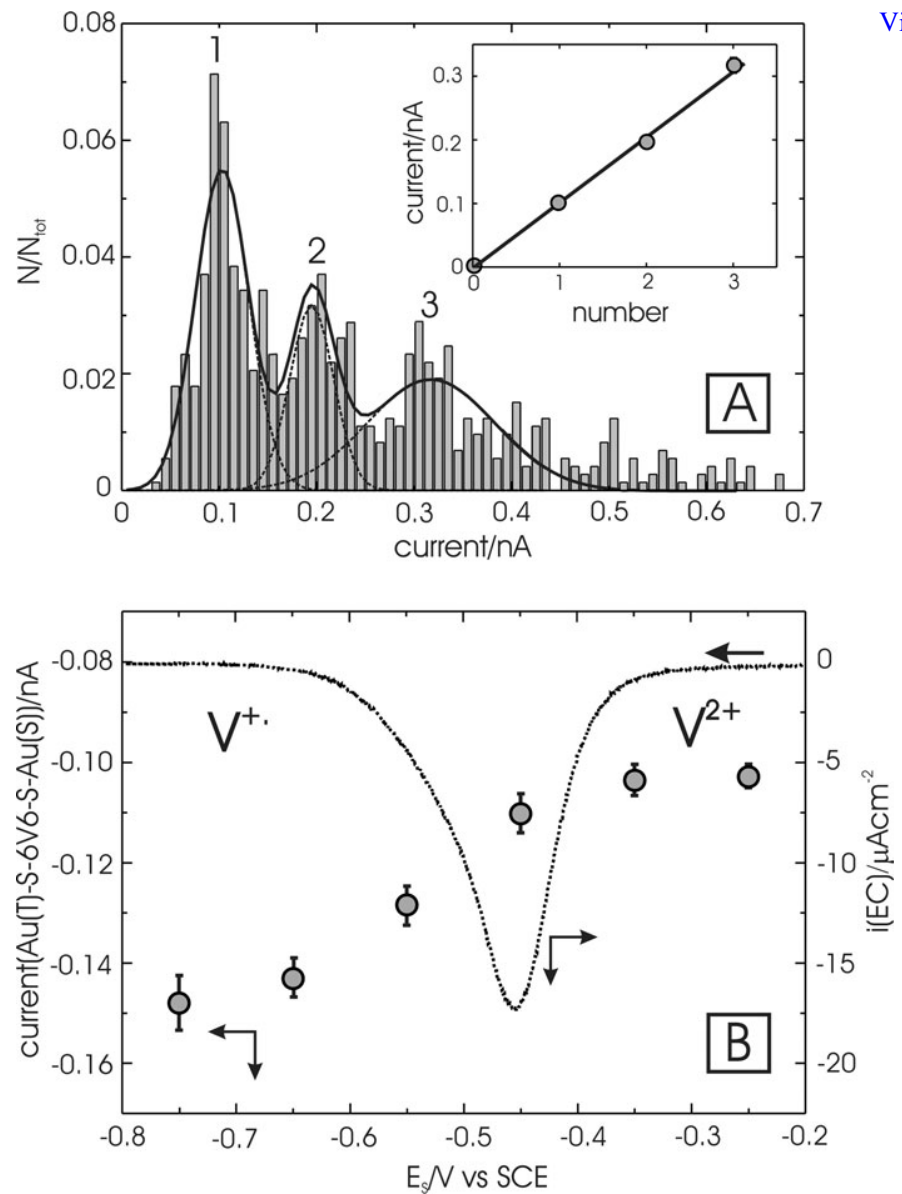

Fig. 8 (A) Histogram constructed from values of the plateau currents of a series of more than 500 individual $i-\Delta z$ pulling curves for molecular junctions $\mathrm{Au}(\mathrm{T})|\mathrm{HS}-6 \mathrm{~V} 6-\mathrm{SH}| \mathrm{Au}(\mathrm{S})$ recorded at $E_{\mathrm{S}}=-0.250 \mathrm{~V}$ and $E_{\mathrm{T}}=-0.150 \mathrm{~V}$. Examples of individual traces were plotted in Fig. 7B. The peaks correspond to 1, 2 or 3 molecules in the gap between two gold electrodes. The peak positions were determined by Gaussian fits (dashed lines) to the histogram peaks (black solid line as the sum of the Gaussians). The inset shows the linear correlation between current and peak number. Fig. 9B represents a plot of the single molecule conductance currents of $\mathrm{Au}(\mathrm{T})|\mathrm{HS}-6 \mathrm{~V} 6-\mathrm{SH}| \mathrm{Au}(\mathrm{S})$ junctions $v s$. the substrate potential $E_{\mathrm{S}}$ at fixed bias $\left(E_{\mathrm{T}}-E_{\mathrm{S}}\right)=0.100 \mathrm{~V}$. The sign convention of the bias voltage gives the negative conduction currents. Each data point was obtained from histograms similar to the one in (A). The dotted line corresponds to the macroscopic current-voltage curve for the reduction of $\mathrm{V}^{2+} \rightarrow \mathrm{V}^{+} \cdot(c f$. cyclic voltammograms in Fig. 1B).

substrate surface ${ }^{25,26}$ This hypothesis is supported by the following observations: (1) Only exponentially decaying current-distance traces were found for the bare supporting electrolyte in $0<i \leq 100 \mathrm{nA}$ ( $c f$. dotted curves in Fig. 7A). (2) No current steps or extended plateaux were detected for the monothiol HS-6V6-H. This molecule contains only one HS-group, and is therefore not capable to form stable molecular bridges between the two gold electrodes. (3) There exist only discrete plateau currents in the $i-\Delta z$ curves (Fig. 7B), which show a distinct dependence on the applied electrode potential (see Fig. 8).

We notice that non-exponential $i-\Delta z$ characteristics were found in 30 to $40 \%$ of all the stretching transients. We attribute this observation to the small electrochemically active area of our coated gold STM tips and to the explicitly chosen low coverage of adsorbed HS-6V6-SH. Inspection of the electrode surface by in situ STM imaging provided a clue to the different nature of the two types of $i-\Delta z$ traces. Individual transients with extended plateaux and rather large current steps $(\Delta i \ll 0.5$ 


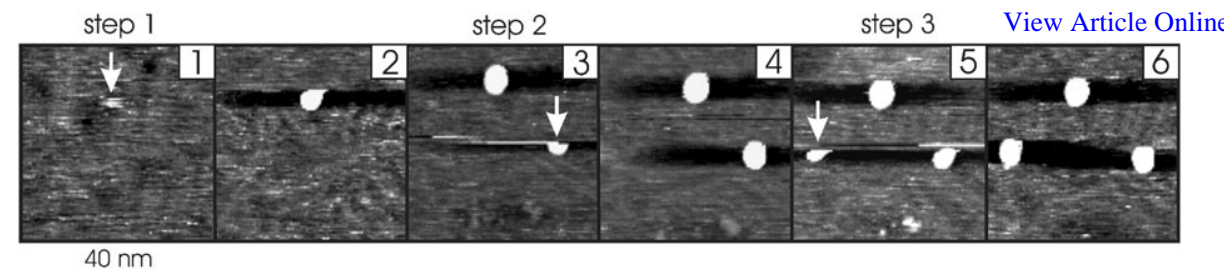

Fig. 9 Series of in situ STM images acquired simultaneously with three successive $i-\Delta z$ traces of type 1 for a disordered adlayer of HS-6V6-SH on $\mathrm{Au}(111)$ in $0.05 \mathrm{M} \mathrm{KClO}_{4}$. The white arrows in the images with odd numbers represent the formation of small gold islands immediately after the completion of an individual pulling event. The experiment was carried out at $E_{\mathrm{S}}=-0.250 \mathrm{~V}$ and $E_{\mathrm{T}}=-0.150 \mathrm{~V}, i_{\mathrm{T}}=100 \mathrm{pA}$. We notice, that the negative potential (and charge density) caused the partial reconstruction of the substrate surface (indicated by corrugations with a double row pattern), which could not be prevented by the low concentration of viologen.

nA) generated typically small gold islands on the $\mathrm{Au}(111)$ surface with a diameter of 3 to $5 \mathrm{~nm}$. Fig. 9 illustrates a characteristic STM sequence acquired simultaneously with the recording of successive $i-\Delta z$ traces of type 1 . The $x-y$ positions of the individual transients are indicated by the arrows in the frames 1, 3 and 5 while the even-numbered frames show the same surface area with the newly created monatomically high gold islands. We observed no indications of a tip crash. We favor the idea that these islands are the result of a substantial material transfer from the gold STM-tip accompanied with the rupture of a large number of local molecular junctions. No evidence of gold islands or of any other morphological changes of the substrate surface were detected for $i-\Delta z$ traces with extended plateaux and gradually decreasing current segments ("type 2") as well as for "type 1" transients with small current steps $(\Delta i \ll 0.5 \mathrm{nA})$.

In an attempt to explore the conductance properties of single molecule junctions Au(T)|HS-6V6$\mathrm{SH} \mid \mathrm{Au}(\mathrm{S})$ we carried out systematic stretching experiments in the low current regime $(i<10 \mathrm{nA})$ at various substrate potential $E_{\mathrm{S}}$ while keeping the bias voltage $\left(E_{\mathrm{T}}-E_{\mathrm{S}}\right)$ fixed. In order to minimize contributions from direct tunneling between tip and substrate we restricted the quantitative analysis to $i<1.0 \mathrm{nA}$. Fig. 7B illustrates, as an example, the low current segments of $i-\Delta z$ stretching transients recorded at $E_{\mathrm{S}}=-0.250 \mathrm{~V}$ and $\left(E_{\mathrm{T}}-E_{\mathrm{S}}\right)=0.100 \mathrm{~V}$. Following a proposal by Haiss et al., ${ }^{26}$ we carried out a statistical analysis of the plateau currents and constructed histograms for substrate potentials in $-0.750 \mathrm{~V} \leq E_{\mathrm{S}} \leq-0.250 \mathrm{~V}$, i.e., within the range of the reduction process $\mathrm{V}^{2+} \leftrightarrow \mathrm{V}^{+} \cdot$. The histograms, which contain data of more than 500 individual transients, show a characteristic series of peaks corresponding to one, two or three molecules in the gap. A typical histogram, which was obtained from data acquired at $E_{\mathrm{S}}=-0.250 \mathrm{~V}$, is plotted in Fig. 8A. Fitting these histograms with Gaussians determines the peak centers and the full width at half maximum. These peak values are then plotted vs. the peak number, and the linear slope yields the current of a single $\mathrm{Au}(\mathrm{T})|\mathrm{HS}-6 \mathrm{~V} 6-\mathrm{SH}| \mathrm{Au}(\mathrm{S})$ junction at fixed bias. We observed linear slopes of the corresponding current-voltage curves for small bias values $\left|E_{\mathrm{T}}-E_{\mathrm{S}}\right| \leq 0.100 \mathrm{~V}$. The resulting single molecule conductance currents are plotted in Fig. $8 \mathrm{~B}$ as a function of $E_{\mathrm{S}}$ at $\left(E_{\mathrm{T}}-E_{\mathrm{S}}\right)=0.100 \mathrm{~V}$. The conductance is rather constant in the stability region of the oxidized viologen dication $\mathrm{V}^{2+}$, increases at $E_{\mathrm{S}}<-0.400 \mathrm{~V}$ and appears to reach a plateau at $E_{\mathrm{S}} \leq-0.700 \mathrm{~V}$, i.e., past the formal potential of the reversible redox process $\mathrm{V}^{2+} \leftrightarrow \mathrm{V}^{+} \cdot$. Attempts to extend the experiment to potentials more negative than $E_{\mathrm{S}}$ gave rather scattered results due to the onset of hydrogen evolution at the chosen $\mathrm{pH}$ of 7 and/or due to the increasing reduction current of trace amounts of oxygen in the system. The change of the single-molecule conductance was also reported by Haiss, ${ }^{26}$ however, no plateau was observed by these authors. The differences between the results of the Liverpool group and those reported in this paper might be attributed to the different electrolytes used and/or to the presence of oxygen which is known to significantly alter the association and redox-properties of dithiol-functionalized viologens. ${ }^{52}$ We tentatively attribute the sigmoidal-type increase of the molecular conductance current to the higher electron density and the higher conjugation of $\mathrm{V}^{+} \cdot$ compared to $\mathrm{V}^{2+}$. This view is supported by force field calculations of Hester et al. ${ }^{78}$ They reported that the inter-ring bond order increases when the viologen dication transforms into the radical upon reduction, which implies that the aromatic character extends across both rings for the latter compound. The values of the single-molecule conductivities of $\mathrm{Au}(\mathrm{T}) \mid \mathrm{HS}-$ 
6V6-SH|Au(S) junctions are higher than those reported for dodecanedithiol (Dite,w0ArQdler(S)),iffe and are comparable with results for 11,11'-bis(4-thiomethylphenyl)-11,11'-diapocarotene $(\mathrm{C}, 2.06 \mathrm{nS}) .{ }^{102}$ All three molecules exhibit approximately the same lengths, and consist of two anchor groups separating an insulating aliphatic chain (DT), a conducting polyene backbone (C) or a dialkyl-viologen unit (this work). The comparison of these three systems clearly demonstrates the sensitivity of the chosen approach to the molecular structure.

Due to the limited number of experimental data points at more negative substrate potentials based on current-distance stretching curves under the present experimental conditions, we postpone the more detailed discussion of possible ET scenarios after reporting results of a second, rather complementary approach, which is also based on an in situ STM configuration.

Asymmetric molecular tunnel junction $\mathbf{A u}(\mathbf{T})-\mathbf{S}-6 \mathrm{~V} 6-\mathrm{H} \mid \mathbf{A u}(\mathbf{S})$. The second approach to explore local electron transfer properties of alkylthiol-terminated viologens is based on a HS-6V6-Hmodified gold nano-electrode, which was inserted as a tip in an in situ STM configuration. We have chosen the monothiol as the redox-active reactant because it is less sensitive to oxygen and chemically more robust than the dithiol. The adsorption on gold electrodes is well defined, and has been carefully investigated by in situ STM and SEIRAS employing macro-electrodes. HS-6V6SH and HS-6V6-H exhibit similar assembly and redox-properties because both contain the functional viologen moiety, two symmetrically arranged alkyl spacer groups of the same length and identical bromide counter ions. The fabrication and characterization of the HS-6V6-SHmodified gold tips have been described before in paragraphs 2.3, 2.5 and 3.2. Pre-selected tips were mounted in the STM setup and stabilized in a carefully controlled argon atmosphere. Contact to the electrochemical STM cell was established under strict potential control of tip and substrate and in the absence of oxygen. The cell was filled with $0.05 \mathrm{M} \mathrm{KClO}_{4}$. The $\mathrm{pH}$ of the electrolyte was adjusted to 7 with $\mathrm{KOH}$ to ensure the chemical integrity of the viologen and to provide a sufficiently wide potential range of ideal polarizability of tip and substrate. The latter was a viologen-free $\mathrm{Au}(111)-(1 \times 1)$ electrode $(c f$. paragraph 3.1$)$. The tip voltammogram $\left(i v s . E_{\mathrm{T}}\right)$ was recorded as a background curve, in the absence of tunneling, with $2 \mathrm{~V} \mathrm{~s}^{-1}$ in $-0.650 \mathrm{~V} \leq E \leq-0.200 \mathrm{~V}$ (dotted

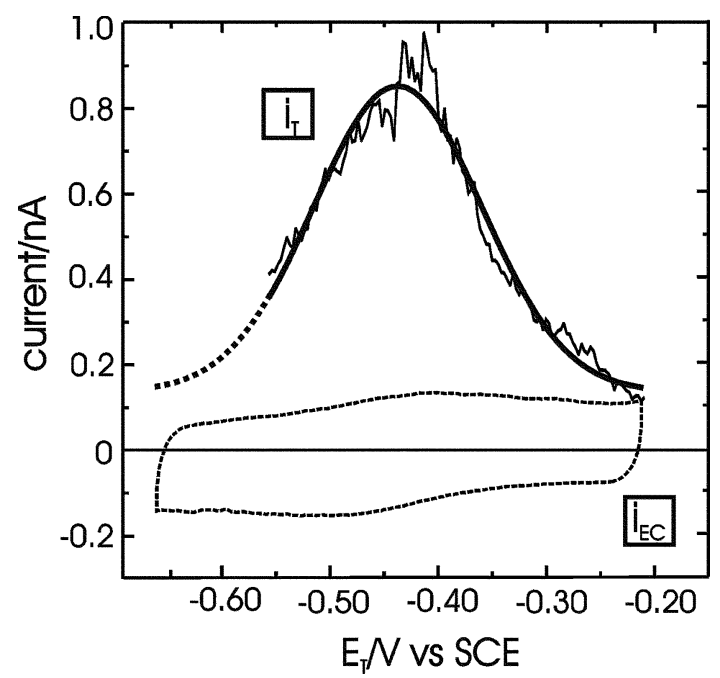

Fig. 10 Tunneling current versus tip potential recorded in $0.05 \mathrm{M} \mathrm{KClO}_{4}$ at $\mathrm{pH} 7$ for an asymmetric tunnel junction $\mathrm{Au}(\mathrm{T})-\mathrm{S}-6 \mathrm{~V} 6-\mathrm{H} \mid \mathrm{Au}(\mathrm{S})$ at fixed bias $\left(E_{\mathrm{T}}-E_{\mathrm{S}}\right)=0.100 \mathrm{~V}$. The solid black trace represents the average of 20 independent half cycles recorded when scanning the tip potential between $-0.650 \mathrm{~V}$ and $-0.200 \mathrm{~V}$ either in positive or in negative direction at $2 \mathrm{~V} \mathrm{~s}^{-1}$, with the feedback being switched off during each individual cycle. The plotted curves are corrected for the electrochemical response of the HS-6V6-H-modified gold tip in the absence of tunneling. This trace is shown as a dashed line. The thick solid and dotted lines represent the result of a Gaussian fit to the redox-molecule-gated tunneling junction. 
line in Fig. 10A). The observed response is determined by (i) the chargeViter butide Quplinthe electrochemical double layer and (ii) the Faraday charge due to reduction and re-oxidation of immobilized HS-6V6-SH. The tunnel junction between the viologen-modified tip and the bare $\mathrm{Au}(111)-(1 \times 1)$ electrode was formed by approaching the tip towards the surface under feedback control until its position was stabilized at a tunneling setpoint current $i_{\mathrm{T}}=100 \mathrm{pA}$. Tip $\left(E_{\mathrm{T}}\right)$ and substrate $\left(E_{\mathrm{S}}\right)$ potentials were adjusted in the stability range of the oxidized viologen $\mathrm{V}^{2+}$. These conditions minimized the interactions between substrate and modified tip. $i_{\mathrm{T}} v s . E_{\mathrm{T}}$ curves were recorded with the feedback switched off during the negative and positive going scans in $-0.650 \mathrm{~V} \leq$ $E \leq-0.150 \mathrm{~V}$ at $2.0 \mathrm{~V} \mathrm{~s}^{-1}$ and at constant bias $\left(E_{\mathrm{T}}-E_{\mathrm{S}}\right)=0.10 \mathrm{~V}$. The latter entails parallel variation of substrate and tip potential relative to a common reference electrode. Both half-cycles exhibit characteristic maxima. After each complete cycle, the feedback was activated again, and the system was given sufficient time to stabilize. An average trace obtained from 20 independent negative and positive half-cycles, which were corrected for the corresponding electrochemical response of the gold tip in the absence of tunneling, is plotted in Fig. 10. The curve shows a welldefined maximum $i_{\mathrm{M}}=(0.89 \pm 0.10) \mathrm{nA}$ at $E_{\mathrm{M}}=-(0.44 \pm 0.02) \mathrm{V}$, which is close to the equilibrium potential of the one-electron redox process $\mathrm{V}^{2+} \leftrightarrow \mathrm{V}^{+}$(paragraphs 3.1. and 3.2.). $i_{\mathrm{T}}$ decreases significantly at $\left|E_{\mathrm{S}}-E_{\mathrm{M}}\right|>0.10 \mathrm{~V}$ and approaches the initially preset tunneling current of $100 \mathrm{pA}$. This trend illustrates the sufficient stability of the HS-6V6-H-modified tunnel junction during the sweep experiment. The $i_{\mathrm{T}}-E_{\mathrm{T}}$ characteristics can be modeled by a Gaussian peak function, which gives $0.14 \pm 0.02 \mathrm{~V}$ as the FWHM. The current of the maximum at EM is significantly larger than the electrochemical background signal and can be attributed convincingly to an enhanced tunneling response. Control experiments with bare gold tips and tips modified with inactive dodecanethiol did not produce these features. We assume that the main contribution to the enhanced tunnel signal originates from one or a small number of redox-active molecules since only few of them will protrude deeper into the gap than others. Since the tunneling current decreases exponentially with distance, the contributions of molecules adsorbed further away from the tip apex are expected to be negligible small.

The appearance of the maximum in the tunnel current can be rationalized as follows: ${ }^{21}$ The formation of a junction $\mathrm{Au}(\mathrm{T})-\mathrm{S}-6 \mathrm{~V} 6-\mathrm{H} \mid \mathrm{Au}(\mathrm{S})$ implies that a molecule with discrete electronic levels is located in the gap between substrate and tip, both representing continuous distributions of electronic levels. The localized electronic states are broadened by interactions of the redox-center with the surrounding electrolyte ${ }^{103,104}$ and with the substrate. ${ }^{105}$ The interaction with the substrate shall be rather small due to the alkyl-spacer unit $\left(\mathrm{C}_{6}\right)$, which spatially separates the $\mathrm{SH}$-anchor group and the redox-active viologen moiety. The broadening introduced by interactions with the solvent arises from the fluctuations of the polar molecules that constantly shift the energy level of the redox center via changing the potential experienced by the electrons in the redox-center. However, the single-electron transfer reorganization Gibbs free energy $\lambda_{\mathrm{R}}$ in the tunneling gap is thought to be significantly smaller than in the semi-infinite space in "classical electron transfer". ${ }^{21}$ As the redox level is exposed to part of the potential drop $\left(E_{\mathrm{T}}-E_{\mathrm{S}}\right)$ a maximum is expected in the current-voltage characteristics. Variation of the overpotential at fixed bias $\left(E_{\mathrm{T}}-E_{\mathrm{S}}\right)$ is equivalent to a parallel shift of the Fermi levels of tip and substrate. The current first rises as the redox level approaches one of the Fermi levels (here the substrate Fermi level). Further increase leads to the current decrease as vacant levels of the positively biased electrode become increasingly thermally inaccessible. These expectations apply if $\mathrm{e} \cdot\left(E_{\mathrm{T}}-E_{\mathrm{S}}\right) \leq \lambda_{\mathrm{R}}$. The position of the tunneling current maximum provides criteria to distinguish between different mechanisms of electron transfer (ET) ${ }^{19,21,43-45}$ Resonance tunneling ${ }^{19,44}$ and a mechanism based on coherent two-step ET ${ }^{21,43}$ predict a maximum in the tunneling current, but in both cases it is expected to be shifted from the equilibrium potential by the reorganization Gibbs free energy $\lambda_{\mathrm{R}}$. A maximum located exactly at the equilibrium potential is predicted for a sequential two-step ET if the bias potential drop at the site of the redox-center is close to half the total potential drop $\left(E_{\mathrm{T}}-E_{\mathrm{S}}\right){ }^{45}$ This theoretical treatment considers the ET in a redox-molecule functionalized tunneling junction as two consecutive interfacial single ET steps with vibration relaxation between the steps. The process comprises a cycle of consecutive molecular reduction and re-oxidation. A particularly interesting feature in the fully adiabatic limit is that each oxidation/reduction cycle is composed of manifolds of interfacial ET events at both electrodes enhancing the electron tunneling significantly, compared to the single ET. 


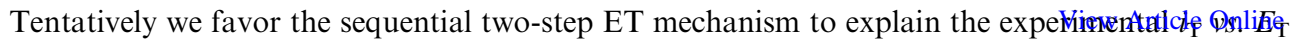
characteristics of the $\mathrm{Au}(\mathrm{T})-\mathrm{S}-6 \mathrm{~V} 6-\mathrm{H} \mid \mathrm{Au}(\mathrm{S})$ tunnel junction. Experiments are in progress to systematically modify composition and structure of the redox-molecule functionalized tunneling junction.

Comparing the results of the current-distance characteristics recorded in stretching experiments with preformed symmetric $\mathrm{Au}(\mathrm{T})|\mathrm{HS}-6 \mathrm{~V} 6-\mathrm{SH}| \mathrm{Au}(\mathrm{S})$ junctions with the current-voltage characteristics of asymmetric $\mathrm{Au}(\mathrm{T})-\mathrm{S}-6 \mathrm{~V} 6-\mathrm{H} \mid \mathrm{Au}(\mathrm{S})$ junctions we may conclude that the former experiment involves dominant contributions from electron transport through the "molecular skeleton". The result of the second experimental approach yielded an enhanced tunneling response due to the activation of a molecular redox state, which appears to be strongly coupled to environmental (solvent) influences. Considerable experimental and theoretical efforts are anticipated to develop a more detailed understanding on the complementary nature of these two experimental approaches for addressing the local molecular redox-activity in an electrochemical environment.

\section{Summary and conclusions}

(1) Two redox-active viologen derivatives, $N$-hexyl- $N^{\prime}$-(6-thiohexyl)-4,4'-bipyridinium bromide (HS-6V6-H) and $N, N^{\prime}$-bis(6-thiohexyl)-4,4'-bipyridinium bromide (HS-6V6-SH), were synthesized based on a modification of literature procedures. The compounds were obtained in very high purity at good yields.

(2) The self-assembly and redox-properties of HS-6V6-H and HS-6V6-SH, immobilized on $\mathrm{Au}(111)-(1 \times 1)$ macro-electrodes and $\mathrm{Au}($ poly) nano-electrodes were investigated by cyclic voltammetry, surface enhanced infrared spectroscopy (SEIRAS) and in situ scanning tunneling microscopy (STM). The strict elimination of the oxygen exposure during the assembly and characterization steps enabled us to identify three distinct types of adlayers for both viologens: a low coverage disordered and a low coverage ordered "striped" phase of flat oriented molecules, and a high coverage monolayer composed of tilted viologen moieties. Using the primitive gold lattice as a basis one could represent the commensurate striped phases in matrix notation as $\left(\begin{array}{cc}18 & 1 \\ -2 & 4\end{array}\right)$ (HS-6V6-H) and $\left(\begin{array}{cc}18 & 1 \\ -1 & 2\end{array}\right)($ HS-6V6-SH), respectively.

(3) The ATR-SEIRAS experiments revealed that the one-electron reduction $\mathrm{V}^{2+} \rightarrow \mathrm{V}^{+} \cdot$ yields, under our experimental conditions, preferentially the radical cation monomer $\left(\mathrm{V}^{+} \cdot\right.$ ) for HS-6V6-H, while a substantial amount of the radical cation dimer $\left(\mathrm{V}^{+} \cdot\right)_{2}$ was observed with (HS-6V6-SH).

The viologen adlayers appear to be stabilized by co-adsorption of $\mathrm{ClO}_{4}{ }^{-}$, which replace $\mathrm{Br}^{-}$ions upon exposure of the modified gold electrodes to an aqueous electrolyte containing $0.05 \mathrm{M} \mathrm{KClO}_{4}$.

(4) An in situ STM configuration was employed to explore electron transport properties of single molecule junctions $\mathrm{Au}(\mathrm{T})|\mathrm{HS}-6 \mathrm{~V} 6-\mathrm{SH}(\mathrm{HS}-6 \mathrm{~V} 6-\mathrm{H})| \mathrm{Au}(\mathrm{S})$ based on analyzing current-distance stretching characteristics. The observed sigmoidal potential dependence of the single molecule conductance, measured at variable substrate potential $E_{\mathrm{S}}$ and at constant bias voltage $\left(E_{\mathrm{T}}-E_{\mathrm{S}}\right)$, was attributed to electronic structure changes of the viologen moiety during the one-electron reduction/re-oxidation process $\mathrm{V}^{2+} \leftrightarrow \mathrm{V}^{+} \cdot$. The simultaneous recording of in situ STM images and successive $i-\Delta z$ traces revealed structural insight into the nature of the "pulling" experiment. Comparison with literature data shows that single molecular conductivities, as determined in $\mathrm{Au}(\mathrm{T}) \mid \mathrm{HS}$-bridge-SH $\mid \mathrm{Au}(\mathrm{S})$ junctions, demonstrate convincingly a molecular structure dependence of the measured signals.

(5) Tunneling experiments in asymmetric, STM-based junctions $\mathrm{Au}(\mathrm{T})-\mathrm{S}-6 \mathrm{~V} 6-\mathrm{H} \mid \mathrm{Au}(\mathrm{S})$ resulted in current $\left(i_{\mathrm{T}}\right)$-voltage $\left(E_{\mathrm{T}}\right)$ curves with a well-defined maximum located at the equilibrium potential of the redox-process $\mathrm{V}^{2+} \leftrightarrow \mathrm{V}^{+} \cdot$, where the surface concentrations of the oxidized and of the reduced form of the reactants are equal. The experimental $i_{\mathrm{T}}-E_{\mathrm{T}}$ characteristics of the HS-6V6-Hmodified tunneling junction were tentatively attributed to a sequential two-step electron transfer mechanism. This experimental result opens up a fascinating approach to explore local electron transfer, and will hopefully inspire further experimental and theoretical activities.

\section{Acknowledgements}

B. H., Z. L. and Th. W. acknowledge support of IFMIT, of the Volkswagen Foundation under contract I 77-116, and of the Research Center Jülich. G. M. thanks the German Academic 


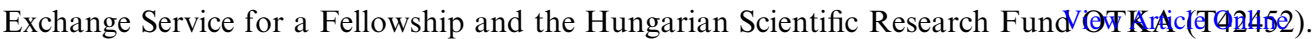
The work of A. B. and M. M. was supported by the German Ministry of Education and Research within the network project MOLMEM (BMBF-FZK 13 N 8360).

\section{References}

1 J. D. Meindel, Q. Chen and J. A. Davis, Science, 2001, 293, 2044.

2 C. Joachim, J. K. Gimzewski and A. Aviram, Nature, 2000, 408, 541.

3 D. L. Klein, R. Roth, A. K. L. Lim, A. P. Alivisatos and P. L. McEuen, Nature, 1997, 389, 699.

4 J. Hu, T. W. Odom and C. M. Lieber, Acc. Chem. Res., 1999, 32, 435.

5 A. Aviram and M. Ratner, Chem. Phys. Lett., 1974, 29, 277.

6 (a) R. Metzger, J. Solid. State Chem., 2002, 168, 696; (b) R. Metzger, Acc. Chem. Res., 1999, $32,950$.

7 Molecular Electronics II, ed. A. Aviram, M. Ratner and V. Mujica, Ann. N.Y. Acad. Sci., New York, 2002, p. 960.

8 M. A. Reed, C. Zhou, C. Muller, T. P. Burgin and J. M. Tour, Science, 1997, 278, 252.

9 C. Kergueris, J. P. Bourgoin, S. Palacin, D. Esteve, C. Urbina, M. Magoga and C. Joachim, Phys. Rev. B, 1999, 59, 12505.

10 (a) J. Reichert, R. Ochs, D. Beckmann, H. B. Weber, M. Mayor and H. v. Löhneysen, Phys. Rev. Lett., 2002, 88, 176804; (b) J. Reichert, H. B. Weber, M. Mayor and H. v. Löhneysen, Appl. Phys. Lett., 2003, 82, 4137.

11 J. Chen, M. A. Reed, A. M. Rawlett and J. M. Tour, Science, 1999, 286, 1550.

12 A. J. Storm, D. van den Broek and S. Lemay, Biophys. J., 2002, 82, 256 P 2.

13 J. G. Kushmerick, D. B. Holt, J. C. Yang, J. Narici, M. H. Moore and R. Shashiidhar, Phys. Rev. Lett., 2002, 89, 86801 .

14 J. O. Lee, G. Lientsching, F. Wiertz, M. Strujik, R. A. J. Janssen, R. Egberink, D. A. Reinhoudt, P. Hadley and C. Dekker, Nano Lett., 2003, 2, 113.

15 E. Tran, M. A. Rampi and G. M. Whitesides, Angew. Chem., Int. Ed., 2004, 43, 3835.

16 Z. Donnhauser, B. A. Mantooth, K. F. Kelley, L. A. Bumm, J. D. Monnell, J. J. Stapleton, D. W. Price, D. L. Allara, J. M. Tour and P. S. Weis, Science, 2001, 292, 2303.

17 S. G. Lemay, J. W. Jansen, M. van der Hout, M. Mooij, M. Bronikowski, P. A. Willis, R. E. Smalley, L. P. Kouwenhoven and C. Dekker, Nature, 2001, 412, 6847.

18 (a) X. Lu, K. W. Hipps, X. D. Wang and U. Mazur, J. Am. Chem. Soc., 1996, 118, 7197; (b) Lscudiero, D. E. Barlow and K. W. Hipps, J. Phys. Chem. B., 2000, 104, 11899; (c) K. Lscudiero, D. E. Barlow and K. W. Hipps, J. Am. Chem. Soc., 2001, 123, 4073; (d) K. Lscudiero, D. E. Barlow and K. W. Hipps, J. Phys. Chem. B, 2002, 106, 996.

19 N. J. Tao, Phys. Rev. Lett., 1996, 76, 4066.

20 D. I. Gittins, D. Bethell, D. J. Schiffrin and R. J. Nichols, Nature, 2000, 408, 67.

21 J. Zhang, Q. Chi, A. M. Kuznetsov, A. G. Hansen, H. Wackerbarth, H. E. M. Christensen, J. E. T. Andersen and J. Ulstrup, J. Phys. Chem. B, 2002, 106, 1131.

22 (a) D. J. Wold and C. D. Frisbie, J. Am. Chem. Soc., 2001, 123, 5549; (b) D. J. Wold, R. Haag, M. A. Rampi and C. D. Frisbie, J. Phys. Chem. B, 2002, 106, 2813.

23 (a) X. D. Cui, A. Primak, X. Zarate, J. Tomfohr, O. F. Sankey, A. L. Moore, T. A. Moore, D. Gust, G. Harris and S. M. Lindsay, Science, 2001, 294, 571; (b) X. D. Cui, X. Zarate, J. Tomfohr, O. F. Sankey, A. Primak, A. L. Moore, T. A. Moore, D. Gust, G. Harris and S. M. Lindsay, Nanotechnology, 2002, $13,5$.

24 F. R. Fan, J. Yang, L. Cai, D. W. Price, S. M. Dirk, D. V. Kosynkin, Y. Yao, A. M. Rawlett, J. M. Tour and A. J. Bard, J. Am. Chem. Soc., 2002, 124, 5550.

25 B. Xu and N. J. Tao, Science, 2003, 301, 1221.

26 W. Haiss, H. van Zalinge, S. J. Higgins, D. Bethell, H. Hörbenreich, D. J. Schiffrin and R. J. Nichols, J. Am. Chem. Soc., 2003, 125, 15294.

27 W. Haiss, R. J. Nichols, H. v. Zalinge, S. J. Higgins, D. Bethell and D. J. Schiffrin, Phys. Chem. Chem. Phys., 2004, 6, 4330.

28 F. Chen, J. He, C. Nuckolis, T. Roberts, J. F. Klare and S. Lindsay, Nano Lett., 2005, 5, 563.

29 J. Chen, M. A. Reed, A. M. Rawlett and J. M. Tour, Science, 1999, 286, 1550.

30 J. Park, A. N. Pasupathy, J. I. Goldsmith, C. Chang, Y. Yaish, J. R. Petta, M. Rinkowski, J. Sithna, H. D. Abruna, P. M. McEuen and D. C. Ralph, Nature, 2002, 407, 722.

31 S. Kubatkin, A. Danilov, M. Hjort, J. Cornil, J. L. Bredas, N. Suhr-Hansen, P. Hedegard and Th. Bjornholm, Nature, 2003, 425, 698.

32 N. B. Zhitenev, H. Meng and Z. Bao, Phys. Rev. Lett., 2002, 88, 226801.

33 R. Rinaldi and R. Cingolani, Physica E, 2004, 21, 45.

34 Y. Luo, C. P. Collier, J. O. Jeppesen, K. A. Nielswen, E. Delonno, G. Ho, J. Perkins, H. R. Tseng, T. Yamamoto, J. F. Stoddart and J. R. Heath, ChemPhysChem, 2002, 3, 519.

35 X. Duan, Y. Huang and C. M. Liber, Nano Lett., 2002, 2, 487.

36 C. Li, W. Fan, D. A. Strauss, B. Lei, S. Asano, D. Zhang, J. Han, M. Meyyappan and Ch. Zhou, J. Am. Chem. Soc., 2004, 126, 7750. 
37 S. Yoshimoto, A. Tada, K. Suto, R. Narita and K. Itaya, Langmuir, 2003, 19, 672. View Article Online

S. Yoshimoto, A. Tada, K. Suto and K. Itaya, J. Phys. Chem. B, 2003, 107, 5836

Mazur and K. W. Hipps, J. Phys. Chem. A, 1999, 103, 9721.

40 A. Alessandrini, M. Gerunda, G. W. Canters, M. P. Verbeet and P. Facci, Chem. Phys. Lett., 2003, 376, 625.

41 J. J. Davis, Philos. Trans. R. Soc. London, Ser. A, 2003, 361, 2807.

42 H. He, J. Zhu, N. J. Tao, L. A. Nagahara, L. Amlani and R. Tsui, J. Am. Chem. Soc., 2001, 123, 7730.

43 A. M. Kuznetsov and J. Ulstrup, Electrochim. Acta, 2000, 45, 2339.

44 W. Schmickler and N. J. Tao, Electrochim. Acta, 1997, 42, 2809.

45 (a) A. M. Kuznetsov and J. Ulstrup, J. Phys. Chem. A, 2000, 104, 11531; (b) A. M. Kuznetsov and J. Ulstrup, Probe Microsc., 2001, 2, 187.

46 M. Krüger, M. R. Buitelaar, T. Nussbaumer, C. Schönenberger and L. Forro, Appl. Phys. Lett., 2001, 78, 1291.

47 S. Rosenblatt, Y. Yaishi, J. Park, J. Gore, V. Sazonova and P. L. McEuen, Nano Lett., $2002,2,869$.

48 M. Di Ventra, N. D. Lang and S. T. Pantelides, Chem. Phys., 2002, 281, 189.

49 Th. Wandlowski, K. Ataka, S. Pronkin and D. Diesing, Electrochim. Acta, 2004, 49, 1233.

50 G. Nagy and Th. Wandlowski, Langmuir, 2003, 19, 10271.

51 C. L. Bird and A. T. Kuhn, Chem. Soc. Rev., 1981, 10, 49.

52 P. M. S. Monk, The Viologens, John Wiley \& Sons, Chichester, 1998.

53 (a) H. C. DeLong and D. A. Buttry, Langmuir, 1990, 6, 1319; (b) H. C. DeLong and D. A. Buttry, Langmuir, 1992, 8, 2491.

54 S. L. Hiley and D. A. Buttry, Colloids Surf., A, 1994, 84, 129.

55 J. Li, G. Chen and S. Dong, Thin Solid Films, 1997, 293, 200.

56 S. A. John and T. Ohsaka, J. Electroanal. Chem., 1999, 477, 52.

57 T. Sagara, N. Kaba, M. Komatsu, M. Uchida and N. Nakashima, Electrochim. Acta, 1998, 43, 2183.

58 T. Sagara, H. Tsuruta and N. Nakashima, J. Electroanal. Chem., 2001, 500, 255.

59 T. Sagara, H. Maeda, Y. Yuan and N. Nakashima, Langmuir, 1999, 15, 3823.

60 R. J. Alvarado, J. Mukkerjee, E. J. Pacsial, D. Alexander and F. M. Raymo, J. Phys. Chem. B, 2005, 109(13), 6164.

61 F. M. Raymo, R. J. Alvarado and E. J. Pacsial, J. Supramol. Chem., 2002, 2, 63.

62 X. Tang, Th. Chneider and D. Buttry, Langmuir, 1994, 10, 2235.

63 W. Haiss, H. v. Zalinge, H. Hörbenreich, D. Bethell, D. J. S. Chiffrin, S. J. Higgins and R. J. Nichols, Langmuir, 2004, 20, 7694.

64 J. L. Hartwell and M. A. Pogorelskin, J. Am. Chem. Soc., 1950, 72, 2040-2044.

65 D. I. Gittins, D. Bethell, R. J. Nichols and D. J. Schiffrin, J. Mater. Chem., 2000, 10, 79-83.

66 A. J. Pearson and J. J. Hwang, J. Org. Chem., 2000, 65, 3466-3472.

67 M. H. Hölzle, Th. Wandlowski and D. M. Kolb, J. Electroanal. Chem., 1995, 394, 271.

68 B. Ren, G. Piccardi and B. Pettinger, Rev. Sci. Instrum., 2004, 75, 837.

69 G. Meszaros, I. Pobelov, Z. Li and Th. Wandlowski, 2005, in preparation.

70 T. Pajkossy, Th. Wandlowski and D. M. Kolb, J. Electroanal. Chem., 1996, 414, 209.

71 C. A. Widrig, C. Chung and M. D. Porter, J. Electroanal. Chem., 1991, 335, 310.

72 A. J. Bard and L. Faulkner, Electrochemical Methods, Wiley, New York, 1980.

73 J. H. Russell and S. C. Wallwork, Acta. Crystallogr., Sect. B. Struct. Crystallogr. Cryst. Chem., 1972, B28, 1527.

74 I. Yu. Polishchuk, L. G. Grineva, A. P. Polishchuk and A. N. Chernega, Zh. Obshch. Khim., 1996, 66, 1530 .

75 A. Ulman, Chem. Rev., 1996, 96, 1533.

76 G. Meszaros, I. Pobelov, B. Han, Z. Li and Th. Wandlowski, 2005, unpublished.

77 M. J. Esplandiu, H. Hagenstrom and D. M. Kolb, Langmuir, 2001, 17, 828.

78 R. E. Hester and S. Suzuki, J. Phys. Chem., 1982, 86, 4626.

79 S. Ghostal, T. Lu, Q. Feng and T. M. Cotton, Spectroelectrochim. Acta, 1988, 44A, 651.

80 S. H. Brienne, R. P. Cooney and G. A. Bowmaker, J. Chem. Soc., Faraday Trans., 1991, 87, 1355.

81 M. Ito, H. Sasaki and M. Takahashi, J. Phys. Chem., 1987, 91, 3932.

82 M. Osawa and K. Yoshi, Appl. Spectrosc., 1997, 51, 512.

83 K. Arihara and F. Kitamura, J. Electroanal. Chem., 2003, 550-551, 149.

84 M. D. Porter, Th. B. Bright, D. L. Allara and C. E. D. Chidsey, J. Am. Chem. Soc., 1987, 109, 3559.

85 H. T. Rong, S. Frey, Y. J. Yang, M. Zharnikov, M. Buck, M. Wühn, Ch. Wöll and G. Helmchen, Langmuir, 2001, 12, 1582.

86 A. Ulman and R. P. Scaringe, Langmuir, 1992, 8, 894.

87 B. Han, Z. Li and Th. Wandlowski, 2005, in preparation.

88 G. E. Poirier, Langmuir, 1997, 13, 2019.

89 M. Böhringer, K. Morgenstern, W. D. Schneider, R. Berndt, F. Mauri, A. de Vita and R. Car, Phys. Rev. Lett., 1999, 83, 324.

90 G. E. Poirier, Langmuir, 1999, 15, 1167.

91 R. Staub, M. Toerker, T. Fritz, T. Schmitz-Hübsch, F. Sellam and K. Leo, Langmuir, 1998, $14,6693$. 
92 T. Y. B. Leung, M. C. Gerstenberg, D. J. Lavrich, G. Scoles, F. Schreiber and G. EieBoikiarcle@mglimair, 2000, 16, 549.

93 T. Y. B. Leung, P. Schwarz, G. Scoles, F. Schreiber and A. Ulman, Surf. Sci., 2000, 458, 34.

94 L. Duan and S. J. Garnett, Langmuir, 2001, 17, 2986.

95 G. Yang and G. Liu, J. Phys. Chem. B, 2003, 107, 8746.

96 D. P. E. Smith, J. K. H. Hörber, G. Binning and H. Nejoh, Nature, 1990, 344, 641.

97 Th. Dretschkow and Th. Wandlowski, Top. Appl. Phys., 2003, 85, 259.

98 S. de Feyter, J. H. van Esch, B. L. Ferringa and F. C. de Schryver, Chem. Eur. J., 2004, $10,1124$.

99 A. Marchenko, S. Lukyanets and J. Cousty, Phys. Rev. B, 2002, 65, 45414-1.

100 S. D. Evan, E. Urankar, A. Ulman and N. Ferris, J. Am. Chem. Soc., 1991, 113, 4121.

101 X. D. Cui, A. Primak, X. Zarate, J. Tomfohr, O. F. Sankey, A. L. Moore, T. A. Moore, D. Gust, L. A. Nagahara and S. M. Lindsay, J. Phys. Chem. B, 2002, 106, 8609.

102 J. He, F. Chen, J. Li, O. F. Sankey, Y. Terazono, Ch. Herrero, D. Gust, Th. A. Moore, A. L. Gust and S. M. Lindsay, J. Am. Chem. Soc., 2005, 127, 1384.

103 R. A. Marcus, J. Chem. Phys., 1956, 24, 979.

104 H. Gerischer, Z. Phys. Chem., Neue Folge, 1960, 26, 223.

105 D. M. Newns, Phys. Rev., 1969, 178, 1123. 